\title{
Spin of a Multielectron Quantum Dot and Its Interaction with a Neighboring Electron
}

\author{
Filip K. Malinowski, ${ }^{1}$ Frederico Martins, ${ }^{1}$ Thomas B. Smith, ${ }^{2}$ Stephen D. Bartlett, ${ }^{2}$ Andrew C. Doherty, ${ }^{2}$ Peter D. Nissen, ${ }^{1}$ \\ Saeed Fallahi, ${ }^{3}$ Geoffrey C. Gardner, ${ }^{3}$ Michael J. Manfra, ${ }^{3,4}$ Charles M. Marcus, ${ }^{1}$ and Ferdinand Kuemmeth ${ }^{1}$ \\ ${ }^{1}$ Center for Quantum Devices and Station $Q$ Copenhagen, Niels Bohr Institute, \\ University of Copenhagen, 2100 Copenhagen, Denmark \\ ${ }^{2}$ Centre for Engineered Quantum Systems, School of Physics, The University of Sydney, \\ Sydney NSW 2006, Australia \\ ${ }^{3}$ Department of Physics and Astronomy, Station Q Purdue, and Birck Nanotechnology Center, \\ Purdue University, West Lafayette, Indiana 47907, USA \\ ${ }^{4}$ School of Electrical and Computer Engineering, and School of Materials Engineering, \\ Purdue University, West Lafayette, Indiana 47907, USA
}

(Received 27 October 2017; revised manuscript received 29 January 2018; published 21 March 2018)

We investigate the spin of a multielectron GaAs quantum dot in a sequence of nine charge occupancies, by exchange coupling the multielectron dot to a neighboring two-electron double quantum dot. For all nine occupancies, we make use of a leakage spectroscopy technique to reconstruct the spectrum of spin states in the vicinity of the interdot charge transition between a single- and a multielectron quantum dot. In the same regime we also perform time-resolved measurements of coherent exchange oscillations between the singleand multielectron quantum dot. With these measurements, we identify distinct characteristics of the multielectron spin state, depending on whether the dot's occupancy is even or odd. For three out of four even occupancies, we do not observe any exchange interaction with the single quantum dot, indicating a spin-0 ground state. For the one remaining even occupancy, we observe an exchange interaction that we associate with a spin-1 multielectron quantum dot ground state. For all five of the odd occupancies, we observe an exchange interaction associated with a spin- $1 / 2$ ground state. For three of these odd occupancies, we clearly demonstrate that the exchange interaction changes sign in the vicinity of the charge transition. For one of these, the exchange interaction is negative (i.e., triplet preferring) beyond the interdot charge transition, consistent with the observed spin-1 for the next (even) occupancy. Our experimental results are interpreted through the use of a Hubbard model involving two orbitals of the multielectron quantum dot. Allowing for the spin correlation energy (i.e., including a term favoring Hund's rules) and different tunnel coupling to different orbitals, we qualitatively reproduce the measured exchange profiles for all occupancies.

DOI: 10.1103/PhysRevX.8.011045

\section{INTRODUCTION}

Spins in semiconducting nanostructures offer a wide variety of approaches to quantum computing. These include approaches based on gate-defined single-electron quantum dots realized in GaAs/AlGaAs heterostructures [1-7], Si/SiGe quantum wells [8-12], or in MOS nanodevices $[13,14]$, as well as spins localized on crystal defects such as phosphorus donors in silicon $[15,16]$. Along with this range of material choices, spins trapped in quantum

Published by the American Physical Society under the terms of the Creative Commons Attribution 4.0 International license. Further distribution of this work must maintain attribution to the author(s) and the published article's title, journal citation, and DOI.
Subject Areas: Condensed Matter Physics,

Quantum Physics,

Quantum Information

dots offer a myriad of possible qubit encodings, including single-dot [11-13], double-dot [8,9,17,18], and triple-dot $[10,19,20]$ schemes, each with distinct advantages.

In contrast to a large body of experimental work on single qubit devices, there are only a handful of demonstrations of two-qubit entangling operations [2,21-24], despite their necessity for quantum computing. Approaches to twoqubit entangling gates based on direct exchange interaction between neighboring tunnel-coupled quantum dots $[1,22,23]$ offer fast, high-fidelity operation [25,26]. Although exchange plays a prominent role in many theoretical proposals [27-40] (see Ref. [41] for a review on spin-based quantum computing in quantum dots), these approaches require dots that are closely spaced next to each other, which makes fabrication and cross-coupling between qubits a challenge for multiqubit systems $[42,43]$. In contrast, 
approaches based on direct charge dipole-dipole interaction can offer longer ranges, but suffer from weak coupling (and thus slow gate times) and comparatively lower fidelities $[2,21]$. Such dipole-dipole interactions could be mediated by superconducting cavities [44-51], thereby providing a mechanism to couple over even longer ranges, similar to what is commonly used for superconducting qubits. However, the small dipole moments and susceptibility to charge noise make it unclear whether these approaches will lead to improvements in gate speed and fidelity for spin qubits.

An attractive alternative that has recently been proposed [52,53] and demonstrated [54,55] is to base two-qubit coupling on exchange interactions, using an intermediate quantum system as a mediator. This approach makes use of the high speed associated with exchange processes, without the need to arrange quantum dots in direct contact with each other, and is therefore attractive for current fabrication techniques. In particular, a mesoscopic multielectron quantum dot [56-61] could serve as both coupling mediator and spacer $[55,62]$, providing a pathway for scalability to multiqubit systems.

To serve as a mediator and spacer, the multielectron quantum dot needs to fulfill several requirements.

(1) The physical size of the multielectron dot should be such that qubit dots can be spaced by at least a few hundred nanometers. This distance facilitates the fabrication of gate electrodes necessary for qubit control and readout. A large size may also allow the coupling of multiple qubits to the same mediator.

(2) The ground-state spin of the multielectron quantum dot must be well defined, to enable the interaction between qubits without entangling with the mediator [63]. Conceptually, a multielectron quantum dot with a nondegenerate spinless ground state appears to be the most straightforward implementation of such a coupler [52,53].

(3) The level spacing of the multielectron quantum dot and the relevant tunnel couplings must be larger than both the energy of the thermal fluctuations $\left(k_{B} T \approx 10 \mu \mathrm{eV}\right.$ for $T=100 \mathrm{mK}$ ) and the excitation spectrum of the control voltage pulses $(\approx 20 \mu \mathrm{eV}$ for $5 \mathrm{GHz}$ bandwidth). This condition is necessary to guarantee that the mediator will be prepared in the ground state and to avoid its accidental excitation.

(4) The ground-state spin, level spacing, and tunnel coupling of the multielectron quantum dot must be tunable with high yield. These parameters depend on mesoscopic details of the multielectron dot, and hence cannot be easily controlled by the choice of geometry alone.

(5) The strength of the exchange interaction must provide a competitive time scale for two-qubit gates. Taking $100 \mathrm{~ns}$ as an upper target for viable two-qubit gates, this puts a lower bound on the coupling strength of roughly $0.01 \mu \mathrm{eV}$.

In this article, we demonstrate that these requirements can be fulfilled by a multielectron quantum dot (except the final requirement, which we address elsewhere [55]). To do this we investigate a linear array of quantum dots in GaAs and configure gate voltages such that an elongated multielectron quantum dot is populated right next to a twoelectron double quantum dot [Figs. 1(a) and 1(b)]. Our approach is based on the fact that the two-electron spin state of the double dot, which can readily be prepared in a singlet state, is sensitive to any spin exchange processes with the neighboring multielectron dot. By pulsing gate voltages towards the charge transition between the right well of the double dot (also referred to as the middle dot) and the multielectron dot, we can systematically induce such spin exchange processes and detect them by subsequent singleshot readout of the double dot. (One may view the double dot as a singlet-triplet qubit, and the presence of spin exchange processes with the multielectron dot as leakage out of the qubit space.) In this way, the double quantum dot serves as a spin-sensitive probe of the multielectron quantum dot.

By employing this double-dot spin probe technique, we study the properties of the multielectron dot in nine subsequent charge occupancies. We are able to identify even and odd occupancy of the dot, and find the following sequence for the ground-state spin of the multielectron dot: With increasing occupancy of the dot, the ground states form a sequence of alternating spin-0 (even occupancy) and spin-1/2 (odd occupancy) states, interrupted once by a spin-1 ground state for a particular even occupancy.

Moreover, we discover a peculiar behavior of the exchange interaction at the charge transition for the cases of spin-1/2 multielectron-dot ground states. Namely, the exchange interaction changes sign when changing dotdefining gate voltages by only a few millivolt. A Hubbard model that includes two orbitals of the multielectron dot as well as a triplet-preferring spin correlation energy enables us to reproduce the energetics associated with the total spin of the multielectron quantum dot. From that model we derive a "phase diagram" that reveals four regimes with qualitatively distinct energy spectra and associated exchange interaction dependencies.

This article is organized as follows. In Sec. II, we describe in detail the studied sample and the sequences of voltage pulses used to induce interactions between the probe electron in the middle dot and the multielectron quantum dot. In Sec. III, we present the observed sequence of ground states as the occupancy of the multielectron quantum dot is increased one electron at a time. Based on this phenomenology we propose a Hubbard model for the description of the multielectron quantum dot. In Sec. IV, we present the experimental evidence for a spin-0 ground state for three of the studied electron occupancies. Section V contains an in-depth study of the interaction between the probe electron and a spin- $1 / 2$ state of the multielectron quantum dot, for five different electron occupancies. In Sec. VI, we present data supporting the observation of a 


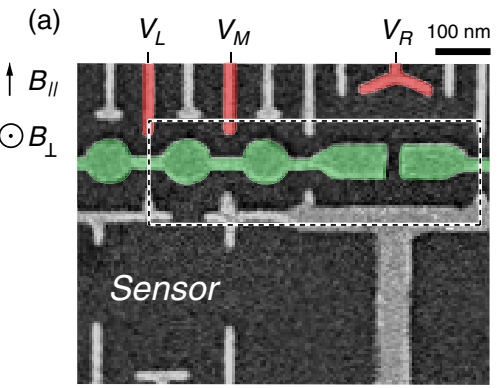

(b)

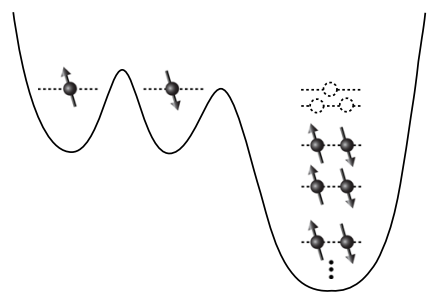

(c)

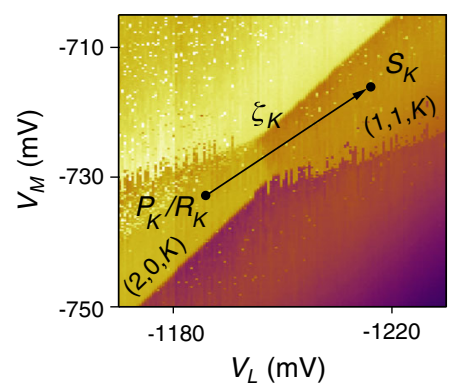

(d)

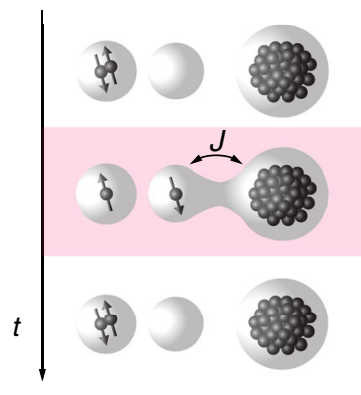



(e)

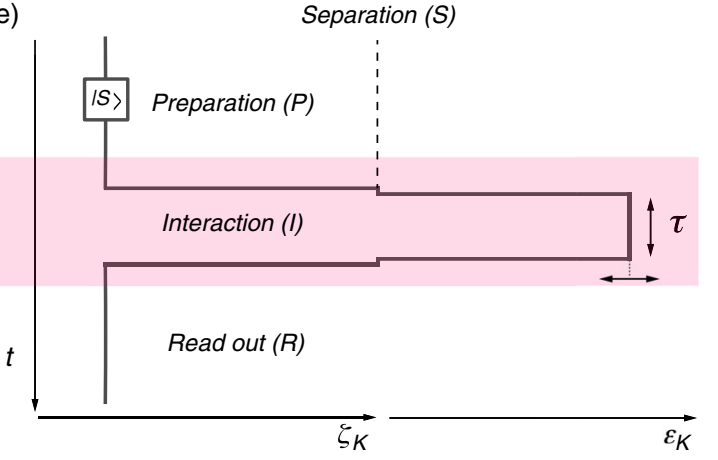

FIG. 1. (a) Scanning-electron micrograph of the device, colored in gray and red to indicate metallic gate electrodes that deplete the 2DEG below the surface (negative voltages). Accumulation gates (colored in green, positive voltages) steepen the resulting confining potential of the quantum dots that form underneath. Voltage pulses applied to the gates $V_{L, M, R}$ control individual electrons in the tripledot array on a nanosecond time scale. (b) Illustration of the electron configuration in the resulting triple quantum dot. We refer to the left and middle dot as the double dot, and to the right dot as the multielectron dot. The precise single-particle level structure and occupation number of the right dot determine the spin properties of the multielectron dot, and are the focus of this experiment. (c) Charge diagrams of the triple quantum dot in the absence of voltage pulses. The left-hand panel shows the interdot charge transition of the two-electron double quantum dot. The right-hand panel presents the charge transition at which the middle electron transfers to the multielectron quantum dot, for different initial occupations of the multielectron dot $(K-1, K$, and $K+1)$ depending on $V_{R}$. Labels $P_{K}, R_{K}$, and $S_{K}$ indicate positions in gate-voltage space at which the electron pair is, respectively, prepared, read out, and separated, if appropriate timedependent voltage pulses are applied. In particular, arrows labeled $\zeta_{K}$ and $\varepsilon_{K}$ indicate axes in gate-voltage space used to define the voltage pulses in this experiment. For example, gate voltages associated with the interaction step $\left(I_{K}\right)$ are varied systematically (see Sec. II), but always remain on the $\zeta_{K}$ or $\varepsilon_{K}$ axis. (d) Operating principle of probing the multielectron spin state by the two-electron double dot. First, a pair of electrons is prepared in a singlet state on the left dot. Next, one of these electrons is transferred to the middle dot, allowing a spin-sensitive interaction $(J)$ with the multielectron dot. In the last step, the spin of the middle electron is measured relative to the reference electron in the left dot by means of Pauli blockade. (e) Implementation of (d) by voltage pulses along $\zeta_{K}$ and $\varepsilon_{K}$. The outcome of each interaction cycle depends on pulse amplitude $(\varepsilon)$ and duration $(\tau)$, and, crucially, on the occupation and spin of the multielectron dot.

spin-1 ground state. Finally, in Sec. VII, we summarize our results.

\section{EXPERIMENTAL SETUP AND TECHNIQUES}

The quantum dots are defined in a GaAs/AlGaAs twodimensional electron gas (2DEG), with electron density $2.5 \times 10^{15} \mathrm{~m}^{-2}$ and mobility $230 \mathrm{~m}^{2} / \mathrm{V} \mathrm{s}$. The $2 \mathrm{DEG}$ is located $57 \mathrm{~nm}$ below the heterostructure surface. A layer of $\mathrm{HfO}_{2}$ with $10 \mathrm{~nm}$ thickness is deposited on top of the heterostructure, followed by the patterning of gold electrodes by electron-beam and lift-off lithography. The oxide layer has a double purpose: first, it allows the application of negative and positive gate voltages without resulting in large leakage currents that would appear through the
Schottky barrier at the GaAs surface; second, it blocks even minute tunneling events between the gate electrodes and the donor layer in the GaAs heterostructure, which would cause effective charge noise and sample switching behavior [64]. The experiment is performed in a dilution refrigerator with the mixing chamber at $20 \mathrm{mK}$.

A scanning-electron micrograph of the active part of the device is presented in Fig. 1(a). The gray and colored structures are metallic gates that are used to define the quantum dot confining potential. The green colored accumulation gate is operated at a small positive voltage of $+40 \mathrm{mV}$. The remaining gates are operated at negative voltages to deplete the $2 \mathrm{DEG}$ and to tune the device. The accumulation gate in this design was introduced to increase the quantum dot potential depth and to improve the 
tunability of the device. The resulting distance between single-electron dots, nominally $150 \mathrm{~nm}$ center to center, is approximately $30 \%$ smaller than in typical designs without the accumulation gate $[20,21,65]$. The device was located in the bore of a superconducting vector magnet. Except for the data presented in Fig. 13(g), the external magnetic field was always applied in plane of the 2DEG, in the direction indicated in Fig. 1(a).

Under the accumulation gate [indicated in Fig. 1(a) by a dashed rectangle], two single-electron quantum dots next to a multielectron quantum dot are tuned up, as schematically indicated in Fig. 1(b). Based on the 2DEG density and the device geometry (dot size roughly $120 \times 250 \mathrm{~nm}$ ), we estimate the electron occupancy of the multielectron quantum dot to be between 50 and 100. The narrow gap in the accumulation gate allows the application of different voltages to different parts of the accumulation gate, but in this study we apply the same (positive) voltage to both parts. A selected number of depletion gates, labeled $V_{L, M, R}$ and shaded in red, are connected to high-bandwidth coaxial lines in the dilution refrigerator, with an associated rise time of $0.8 \mathrm{~ns}$. To perform submicrosecond charge and spin manipulations, time-varying voltage pulses are applied to these gates, while dc voltages (applied to all gates) can be modified slowly to explore various occupancies and tunings of the multielectron dot.

In Fig. 1(c), we present typical charge stability diagrams of the double-dot multielectron-dot system. On the left we present the charge diagram with respect to gate voltages $V_{L}$ and $V_{M}$. These gate voltages are dedicated to control the state of the double quantum dot. Indeed, this diagram reveals the interdot charge transition of the double dot, occurring between the $(2,0, K)$ and $(1,1, K)$ regions. Here, $(L, M, R)$ indicates the number of electrons in the left, middle, and multielectron dot, respectively, and $K$ is an unknown but fixed integer between 50 and 100, which we can vary by changing the dc tuning of the device. By adjusting the voltages $V_{L}$ and $V_{M}$ in such a way that these gate voltages follow the detuning axis $\zeta_{K}$ [Fig. 1(c), lefthand panel], the charge configuration of the two electrons within the double dot can be controlled without affecting the number of electrons on the multielectron dot.

On the $\zeta_{K}$ axis we define point $P_{K}\left(R_{K}\right)$ that serves as the preparation (readout) point of the double-dot spin state. We also define a separation point $S_{K}$ at which the two electrons within the double dot do not interact via exchange with the multielectron quantum dot and only very weakly interact via exchange with each other. Having chosen the separation point $S_{K}$, we can map out the charge stability diagram of the multielectron dot as a function of voltages $V_{M}$ and $V_{R}$, as illustrated in the right-hand panel of Fig. 1(c). In this charge diagram we identify the point $S_{K}$ in the gate-voltage space, and use it to define the $\varepsilon_{K}$ axis that runs from $S_{K}$ through the interdot charge transition between the $(1,1, K)$ and $(1,0, K+1)$ regions. By controlling the position of gate voltages along this axis (i.e., point $I_{K}$ ), we can induce the interaction between the single electron in the middle dot and the multielectron dot, while preserving the reference electronic spin in the left dot. By slightly changing the dc tuning of the quantum dots (in particular $V_{R}$ ), we can change the occupancy of the multielectron dot one by one, and define analogous control axes for different charge states. In Fig. 1(c) these are schematically illustrated by axes labeled $\varepsilon_{K-1}$ and $\varepsilon_{K+1}$.

Having defined the points $P_{K} / R_{K}, S_{K}$, and detuning axes $\zeta_{K}$ and $\varepsilon_{K}$ for each occupancy $K$ of the multielectron quantum dot, we can apply gate-voltage pulses that quickly change the charge configuration from $(2,0, K)$ to $(1,1, K)$ to $(1,0, K+1)$ and back, thereby allowing the study of interactions between the middle electron with the multielectron quantum dot [illustrated in Figs. 1(d) and 1(e)]. Specifically, the first pulse initiates the system at point $P_{K}$, resulting in a pair of electrons prepared in the singlet state $|S\rangle$ on the leftmost quantum dot. From there, a pulse to point $S_{K}$ separates these two entangled electrons while maintaining their spin-singlet correlation. At point $S_{K}$ we pause for one clock cycle of the waveform generator, which varies between 0.83 and $2.5 \mathrm{~ns}$ in this study. This precaution ensures that we indeed transfer the electron through the middle dot to the multielectron dot, instead of ejecting it into one lead followed by injection of another electron to the multielectron dot from the other lead. Because one clock cycle is shorter than the dephasing time due to interaction with the nuclear spins [66-68], $T_{2}^{*} \approx 10 \mathrm{~ns}$, this waiting time does not significantly affect the singlet correlation of the two electrons. The next step of the pulse cycle jumps to a point along the $\varepsilon_{K}$ axis and remains there for time $\tau$. It is during this stage that the interaction between the electron and multielectron quantum dot occurs. We then return to $S_{K}$ for another clock cycle of the waveform generator. Finally, we pulse back to the $(2,0, K)$ charge configuration at point $P_{K} / R_{K}$. The system reaches this charge configuration only if the pair of electrons on the double quantum dot forms a spin-singlet state, otherwise the system is Pauli blocked and remains in the metastable $(1,1, K)$ charge state. The reflectometry readout of the conductance through the neighboring sensor $\operatorname{dot}\left(V_{\mathrm{RF}}\right)$ allows us to distinguish these charge states, thereby yielding a single-shot spin readout ("singlet" or "triplet") for a reflectometry integration time between 5 and $20 \mu$ s. Both parameters $\varepsilon_{K}$ and $\tau$ are varied within a sequence of pulse cycles. Using this pulsed-gate technique, coherent spin dynamics and incoherent spin mixing can both be detected, by choosing $\tau$ sufficiently short or long, respectively.

\section{MULTIELECTRON QUANTUM DOT}

In this section, we present and discuss a theoretical model that describes the multielectron quantum dot and its tunnel coupling to the double quantum dot. It is used in subsequent sections to understand the experiments that 
systematically induce exchange interactions between the single electron residing in the middle dot and the multielectron quantum dot.

Appropriate for a semiconductor with negligible spinorbit coupling (but allowing for nontrivial electron-electron correlations), we model the multielectron quantum dot with the Hamiltonian:

$$
\hat{H}_{R}=U_{R} \hat{n}_{R}^{2}+\sum_{\substack{\lambda \in \mathbb{N} \\ \alpha=1, \downarrow}} \varepsilon_{\lambda} \hat{c}_{\lambda, \alpha}^{\dagger} \hat{c}_{\lambda, \alpha}-\frac{\xi}{2} \hat{S}^{2}+g \mu_{B} B_{\|} \hat{S}_{z},
$$

where $U_{R}$ is the dot charging energy, $\hat{n}_{R}$ is the operator counting the total number of electrons, $\hat{c}_{\lambda, \alpha}^{(\dagger)}$ are the annihilation (creation) operators for an electron on the single-particle level $\lambda$ with spin $\alpha, \varepsilon_{\lambda}$ are the energies of the single-particle levels, $\hat{S}$ is the total spin operator, $\xi$ is the spin correlation energy, $g=-0.4$ is the electronic $g$ factor, $\mu_{B}$ is the Bohr magneton, $B_{\|}$is the amplitude of the applied magnetic field, and $\hat{S}_{z}$ is the total spin projection on the direction of the magnetic field. The subscript $R$ in this formula refers to the multielectron dot as the right dot, whereas $L$ and $M$ denote the left and middle single-electron dots. The influence of the applied magnetic field on the quantum dot orbitals is neglected in this model.

The relative strength of the three terms present in this Hamiltonian determines the spin properties of the multielectron quantum dot. The charging energy of the multielectron quantum dot, $U_{R} \approx 1 \mathrm{meV}$, is estimated from the distance between the multielectron-dot charge transitions [ $\Delta V_{R} \approx 20 \mathrm{mV}$; Fig. 1(c)] and the typical lever arm between the gates and the dots in devices of similar design $(\approx 0.05 e)$. The charging energy may vary slightly as a function of the dot occupancy, as additional electrons may increase the effective size of the quantum dot (soft confining potential). For the results presented here it is only relevant that the charging energy is much larger than the other energy scales, discussed below.

From the lithographic size of the device we estimate the typical level spacing [69] to be $\langle\Delta E\rangle=\pi \hbar^{2} / m^{*} A \approx$ $0.12 \mathrm{meV}$, where $\hbar$ is the reduced Planck constant, $m^{*}$ is the effective electron mass in GaAs, and $A$ is the area of the two-dimensional quantum dot. However, the lack of symmetry causes the level spacings to vary. The determination of level spacings $\Delta E$ and correlations between them for a particular mesoscopic quantum dot is a formidable theoretical task. Their distributions are typically described using random matrix theory with the orthogonal ensemble [56,59,70-72], which by itself neglects interaction effects. Interaction effects can be introduced by means of randomphase approximation [73], mean-field approximation [72], density-functional theory [74,75], the Anderson model [76], or by an on-site Hubbard interaction term [70] (for a review, see Ref. [60]). Modeling a large twodimensional quantum dot with up to 200 electrons using density-functional theory revealed that the emerging electron-electron interaction effects are substantially stronger than those predicted by subjecting the randomphase approximation to the rules of random matrix theory, evidenced by a relatively large probability for high-spin ground states $(S \geq 1)$ [75].

For nonmesoscopic (elliptical) few-electron dots, recent full configuration interaction calculations indicate that negative exchange energies at zero magnetic field are possible for as few as four electrons in GaAs (and similarly in silicon), depending on the dot's size and ellipticity [77]. The possibility of singlet-triplet inversions, negative exchange, and potential use in spin-based quantum information applications was already pointed out in prior theoretical studies that always invoked orbital coupling to magnetic fields $[28,33,37]$, supported by experimental indications for such orbital-coupling effects in circular and elliptical few-electron dots $[78,79]$.

For the results presented here, we consider the case where the width of the level-spacing distribution $\sigma_{\Delta E}$ is comparable to $\Delta E$ and $\xi$, allowing for the emergence of mesoscopic magnetism already at zero magnetic field $[71,80]$. Also, we assume that the single-particle energies do not depend on the occupancy of the dot, consistent with earlier experiments that found that the excitation spectra of (few) subsequent charge states are highly correlated [58].

The spin correlation energy $\xi$ is the most difficult quantity to estimate due to the lack of data in the literature. This energy favors parallel spin configuration in the case of nearly degenerate orbitals, and in that sense can be viewed as a mesoscopic analogue of Hund's multiplicity rule [81]. We make the assumption that $\xi / 2$ is comparable but smaller than $\langle\Delta E\rangle$, based on two experimental observations. First, we observe no significant polarization of the electronic spins, which would be expected from the Stoner instability for the case $\xi / 2>\langle\Delta E\rangle[60,71,84]$. Second, we observe the occurrence of ground states with spin $>1 / 2$, which indicates that the single-particle energy of the first excited state sometimes becomes smaller than $\xi$. This excludes the possibility $\xi \ll\langle\Delta E\rangle$. Throughout the paper we use $U_{R}=1 \mathrm{meV}$ and $\xi=0.1 \mathrm{meV}$, while we use different $\Delta E$ for different occupancies of the multielectron quantum dot (justified by level filling of a mesoscopic spectrum). This choice is consistent with the occasional observation of spin1 ground states in similar quantum dots with moderate correlation effects (see below).

Our study involves modeling of the interaction between the spin occupying the middle $\operatorname{dot} M$ [Fig. 1(b)] and one or two lowest empty or partially occupied orbitals of the multielectron quantum dot. The occupancy of the multielectron quantum dot will determine the nature of this interaction. We consider three cases, ordered by increasing complexity.

(i) All levels of the multielectron quantum dot are either empty or doubly occupied, and the total spin is zero. In this case, the interaction of the double quantum dot with the 
TABLE I. Summary of the inferred ground-state spin for 9 subsequent charge occupancies of the multielectron quantum dot. A sequence of alternating spin- 0 and spin- $1 / 2$ states is interrupted once by a spin-1 ground state. To emphasize the role of electron parity, we have arbitrarily chosen one even dot occupation as a reference, labeled $2 N$, and specify other dot occupations relative to that occupation.

\begin{tabular}{lcc}
\hline \hline $\begin{array}{l}\text { Multielectron-dot } \\
\text { occupancy }\end{array}$ & $\begin{array}{c}\text { Inferred ground-state } \\
\text { spin }\end{array}$ & $\begin{array}{l}\text { Experimental } \\
\text { evidence }\end{array}$ \\
\hline $2 N-5$ & $1 / 2$ & Fig. 10 \\
$2 N-4$ & 0 & Fig. 4(a) \\
$2 N-3$ & $1 / 2$ & Fig. 7 \\
$2 N-2$ & 0 & Fig. 4(b) \\
$2 N-1$ & $1 / 2$ & Fig. 8 \\
$2 N$ & 0 & Fig. 3 \\
$2 N+1$ & $1 / 2$ & Fig. 9 \\
$2 N+2$ & 1 & Fig. 13 \\
$2 N+3$ & $1 / 2$ & Fig. 11 \\
\hline \hline
\end{tabular}

multielectron quantum dot can be modeled as an effective interaction of the spin of the middle dot $M$ tunnel coupled to one unoccupied orbital in the right dot $R$ (Sec. IV).

(ii) There is exactly one unpaired spin in the multielectron quantum dot, giving rise to a total spin of $1 / 2$ (Sec. V). In this case, the interaction of the double quantum dot with the multielectron quantum dot can be modeled as an effective interaction of the spin of the middle dot $M$ tunnel coupled to a single spin in the right dot $R$. Depending on the details of the spin interaction terms of the multielectron quantum dot, we must consider both the partially occupied orbital of the multielectron quantum dot as well as the lowest unoccupied orbital.

(iii) Several unpaired spins in the multielectron quantum dot form a nonzero total spin, e.g., spin 1 (Sec. VI). This requires the modeling of two closely spaced orbitals in the right dot, $R 1$ and $R 2$, in conjunction with a sufficiently large spin correlation energy $\xi$ relative to the orbitals' spacing $\Delta E$.

In our experimental data, which we discuss in detail in Secs. IV-VI, we find that all three cases do occur as we explore different occupation numbers of the multielectron dot. Table I provides an overview of the observed sequence of alternating spin- 0 and spin- $1 / 2$ ground states. This sequence is interrupted once by a spin-1 ground state instead of spin-0 (with profound implications for the associated exchange profiles). The occurrence of a nonminimal ground-state spin in our experiments corroborates earlier findings in GaAs quantum dots by Folk et al. [59] and Lindemann et al. [85], who identified ground-state spins by studying the change of the Coulomb peak spacings with magnetic field.

\section{SPIN-0 BEHAVIOR FOR EVEN OCCUPANCIES $(K=2 N-4,2 N-2,2 N)$}

We first focus on even occupancies of the multielectron dot, specifically $2 N-4,2 N-2$, and $2 N$, and show

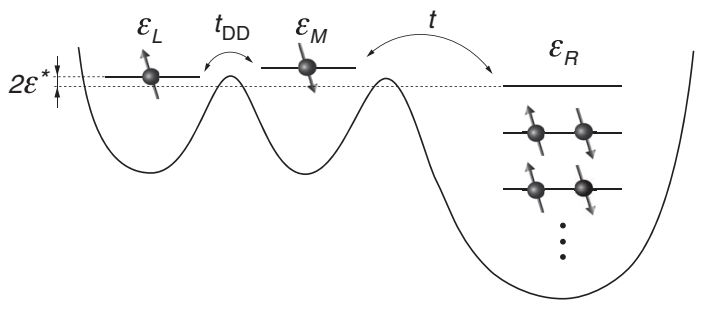

FIG. 2. Schematic of the even-occupied spinless multielectron dot, coupled to the two-electron double dot. Symbols $\varepsilon_{L / M / R}$ indicate the single-particle energies of the lowest orbitals in the double dot and the lowest unoccupied orbital in the multielectron dot. Arrows indicate tunnel couplings within the double dot $\left(t_{\mathrm{DD}}\right)$ and between the middle and the multielectron dot $(t)$. For simulations, the detuning of the right dot relative to the left dot is varied $\left(\varepsilon^{*}\right)$, which allows the generation of energy spectra [as in Fig. 3(b)] and comparison to experimental leakage spectroscopy data [see discussion of Figs. 3(c) and 3(d)].

experimental evidence that these have a spin- 0 ground state. (Here, $2 N$ indicates a specific even number of electrons, estimated to lie between 50 and 100.) Provided that the spin correlation term is smaller than the level spacing between the ground and the first excited state, the model introduced in Sec. III suggests that the ground state of the multielectron dot can be thought of as an effective vacuum state; i.e., all singleparticle states below the Fermi energy are occupied by singlet pairs of electrons [Figs. 2 and 3(a)]. We therefore expect that the double dot will interact with the multielectron dot as if it was an unoccupied dot, and the spin of an electron tunneling into an unoccupied orbital of the multielectron dot would not experience any exchange dynamics. In this sense, the double dot coupled to the multielectron dot with even occupancy should be qualitatively similar to a two-electron triple dot.

We describe this situation using a phenomenological model based on the Hamiltonian for the multielectron dot detailed in Sec. III, augmented by terms for the neighboring tunnel-coupled two-electron double quantum dot. Appropriate for spinless even-occupancy ground states, we neglect orbitals of the multielectron dot below the Fermi energy (these are occupied by spin-singlet electron pairs). We also neglect all but the lowest unoccupied orbital, arriving at a Hubbard model for the three dots, each having a single orbital, labeled $L, M$, and $R$ :

$$
\begin{aligned}
\hat{H}_{\text {spin- } 0}= & \sum_{i=L, M, R}\left(\varepsilon_{i} \hat{n}_{i}+\frac{U_{i}}{2} \hat{n}_{i}\left(\hat{n}_{i}-1\right)\right)+\sum_{i \neq j} \frac{K_{i j}}{2} \hat{n}_{i} \hat{n}_{j} \\
& +\frac{g \mu_{B} B_{\|}}{2}\left(\hat{n}_{\uparrow}-\hat{n}_{\downarrow}\right) \\
& -t_{\mathrm{DD}} \sum_{\alpha=\uparrow, \downarrow}\left(\hat{c}_{L, \alpha}^{\dagger} \hat{c}_{M, \alpha}+\hat{c}_{M, \alpha}^{\dagger} \hat{c}_{L, \alpha}\right) \\
& -t \sum_{\alpha=\uparrow, \downarrow}\left(\hat{c}_{M, \alpha}^{\dagger} \hat{c}_{R, \alpha}+\hat{c}_{R, \alpha}^{\dagger} \hat{c}_{M, \alpha}\right)
\end{aligned}
$$


(a)
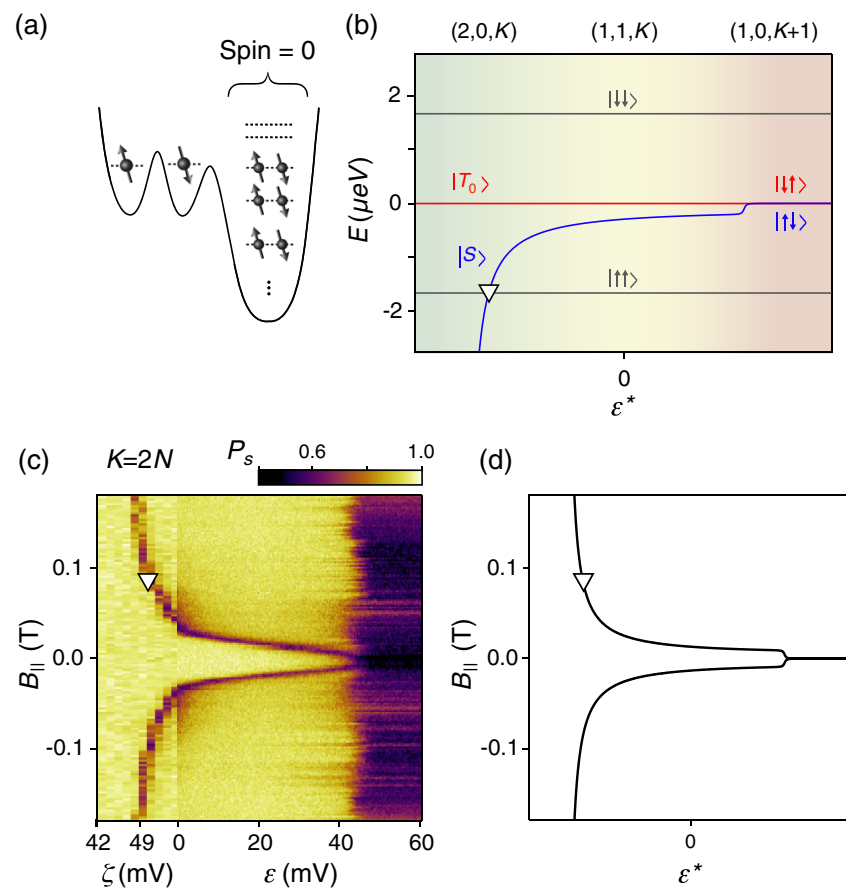

(e)

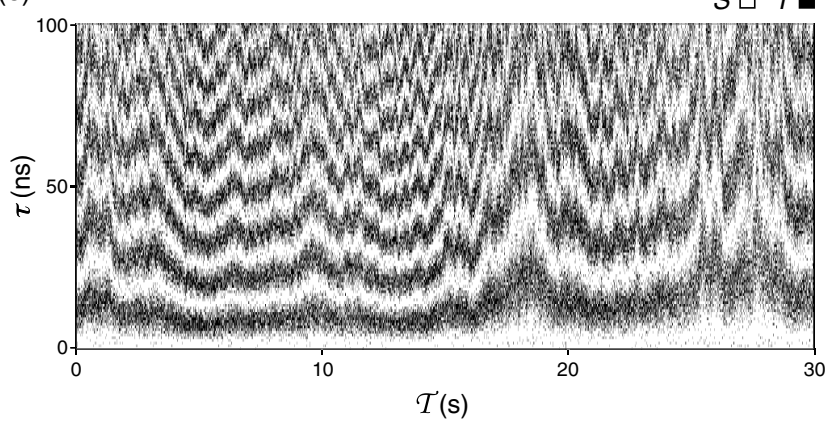

FIG. 3. Spin-0 ground-state behavior of the $2 N$ occupied multielectron dot. (a) Schematic representation of the electron configuration for even-occupied multielectron dot with spin-0 ground state. Electrons below the Fermi energy in the multielectron dot are assumed to pair up into spin singlets. (b) Eigenstates of a two-electron triple dot system for fixed applied magnetic field, calculated as a function of $\varepsilon^{*}$. For suitable input parameters the resulting spin states (labeled) are a useful model for comparison with experimental data obtained from multielectron-dot charge states $(2,0,2 N),(1,1,2 N)$ and $(1,0,2 N+1)$ (see main text). (c) Experimental leakage spectroscopy for $K=2 N$, revealing strong exchange coupling in $(2,0,2 N)$, i.e., within the double dot, and vanishing exchange interaction in $(1,0,2 N+1)$. We associate the sharp feature of suppressed $P_{S}$ (white triangle) with the $S-T_{+}$crossing and the overall suppression of $P_{S}$ above $\varepsilon \approx 45 \mathrm{mV}$ with $S-T_{0}$ oscillations arising from Overhauser gradients. (d) Leakage spectrum expected from the $S-T_{+}$crossing within the Hubbard model (white triangle in panel (b)). (e) Time-resolved measurement of coherent oscillations between $|S\rangle$ and $\left|T_{0}\right\rangle$ two-electron spin states in $(1,0,2 N+1)$ charge state, for $B_{\|}=700 \mathrm{mT}$ and $\varepsilon=60 \mathrm{mV}$. The observed fluctuations of precession frequencies with laboratory time $\mathcal{T}$ are characteristic of fluctuating Overhauser field gradients arising from nuclear spin dynamics within the GaAs sample. where $\hat{n}_{i}$ is the operator counting the number of electrons on each dot. As illustrated in Fig. 2, the term $\varepsilon_{i}$ describes the gate-tunable chemical potential of the left $L$, middle $M$, and the right multielectron $R$ dot. $U_{i}$ and $K_{i j}$ represent, respectively, on- and off-site Coulomb interaction energies. In the second line, describing the Zeeman splitting, $\hat{n}_{\uparrow / \downarrow}$ indicates the number of electrons with spin parallel and antiparallel to the external magnetic field. The third and last line incorporate the tunnel coupling $t_{\mathrm{DD}}$ within the double dot and the tunnel coupling $t$ between the middle and right dot.

This effective Hamiltonian can be solved in the twoelectron configuration to yield the energy of all possible spin states, using input parameters motivated by experiment. For simplicity we plot in Fig. 3(b) the eigenenergies as a function of electrostatic detuning between the right and left dot, $\varepsilon^{*} \equiv\left(\varepsilon_{R}-\varepsilon_{L}\right) / 2$. Qualitatively, the dependence on $\varepsilon^{*}$ (which has units of energy) can be compared with the observed dependence on the experimental detuning parameters $\zeta_{K}$ and $\varepsilon_{K}$ in Fig. 3(c) (which have units of voltage). For the calculated energy spectrum in Fig. 3(b), we used $t=30 \mu \mathrm{eV}$ and $t_{\mathrm{DD}}=15 \mu \mathrm{eV}$. For clarity, we also assumed a finite magnetic field, $B_{\|}=0.07 \mathrm{~T}$, which shifts the energy of the fully polarized spin states (remaining parameters, fixed throughout the paper, are specified in the Appendix). Recall that the "unoccupied" state of the multielectron dot is assumed to be an effective "vacuum" state with $2 N$ electrons in a spin- 0 configuration; i.e., the evolution of charge states from $(2,0,2 N)$ via $(1,1,2 N)$ to $(1,0,2 N+1)$ in the experiment should be compared to the evolution from $(2,0,0)$ via $(1,1,0)$ to $(1,0,1)$ in the model [indicated by the colored background shading in Fig. 3(b)]. Specifically, we are interested in the evolution of the singlet double-dot state $|S\rangle$ and the unpolarized triplet double-dot state $\left|T_{0}\right\rangle$, as their splitting is a witness of exchange effects. Their calculated dependence on $\varepsilon^{*}$ in Fig. 3(b) can be understood as follows. In the $(2,0,2 N)$ charge state (i.e., towards negative $\varepsilon^{*}$ ), the singlet and triplet states of the two-electron double dot are split by the well-known intradot exchange energy. This splitting arises from the Pauli exclusion principle and the finite single-particle level spacing of the left dot (modified by small corrections arising from weak correlation effects), although in our model it diverges because we consider only one orbital in each dot. Towards the $(1,1,2 N)$ tuning, i.e., $\varepsilon^{*}=0$, the exchange splitting gradually decreases as the overlap of the electronic wave functions decreases. Finally, in the $(1,0,2 N+1)$ tuning, the exchange splitting reduces even further, as the two electrons occupy distant dots and interact only via superexchange. In this large- $\varepsilon^{*}$ limit, we assume that superexchange is negligible and label the two degenerate states by basis states $|\uparrow \downarrow\rangle$ and $|\downarrow \uparrow\rangle$ (rather than singlet and triplet), as these states are known to become the correct energy eigenstates if differences between the total effective magnetic field in each dot are taken into account (caused, 
for example, by uncontrolled Overhauser fluctuations, which are omitted in our effective Hamiltonian). In Fig. 3(b), we also label the fully polarized spin states as $|\uparrow \uparrow\rangle$ and $|\downarrow \downarrow\rangle$. For negative $\varepsilon^{*}$ they correspond to the wellknown fully polarized triplet states within a two-electron double-dot system.

Using spin leakage spectroscopy (which can be viewed as an extension of the "spin funnel" measurement of a double quantum dot $[8,66]$ ), we experimentally map out the exchange profile across the three charge configurations $(2,0, K),(1,1, K)$, and $(1,0, K+1)$. By applying the pulse sequence introduced in Sec. II, we prepare the double dot in a singlet state $|S\rangle$, and then pulse to the various interaction points $I_{K}$ along the $\zeta_{K}$ and $\varepsilon_{K}$ axes using a fixed interaction time $\tau$. By choosing $\tau$ sufficiently long (here, $150 \mathrm{~ns}$ ), incoherent mixing between the middle spin and other spin states can be detected, as any such processes reduce the probability $P_{S}$ of detecting a spin-singlet state when pulsing back to the readout configuration of the double dot. We repeat this procedure for various values of the in-plane magnetic field, up to $B_{\|}=200 \mathrm{mT}$, and associate any significant decrease in $P_{S}$ with leakage from the singlet state.

The result is shown in Fig. 3(c) for one particular even occupation of the multielectron dot, $K=2 N$. Clearly, there is a sharp feature of reduced $P_{S}$, marked by a white triangle, that depends on the applied magnetic field. We associate it with the crossing of the singlet state $|S\rangle$ and the fully polarized triplet state $\left|T_{+}\right\rangle=|\uparrow \uparrow\rangle$. (At negative magnetic field, mixing between $|S\rangle$ and $\left|T_{-}\right\rangle=|\downarrow \downarrow\rangle$ causes an analogues feature, leading to a leakage spectrum that is symmetric with respect to $B_{\|}=0$.) Indeed, at such crossings rapid mixing due to uncontrolled Overhauser gradients is expected to occur, changing electronic spin projections by 1 on a time scale of $T_{2}^{*} \approx 10 \mathrm{~ns}$ [66]. This leakage feature diverges to high field in the $(2,0,2 N)$ configuration, indicating that the exchange interaction between the two electrons within the double dot, i.e., the single-particle spacing in the left dot, is relatively large. (Here, we use that for this particular crossing, the associated external magnetic field $B_{\|}$can be converted into energy using the Zeeman shift associated with $|\uparrow \uparrow\rangle$, i.e., $g \mu_{B}\left|B_{\|}\right|$, where $g \approx-0.4$ is the electronic $g$ factor for GaAs and $\mu_{B}$ is the Bohr magneton [66,86].) Towards the $(1,1,2 N)$ configuration the leakage feature gradually moves towards $B_{\|}=0$, indicating a decrease of the exchange interaction strength. Finally, it converges to zero field in the $(1,0,2 N+1)$ configuration, consistent with the two electrons being spatially separated and no longer exchange coupled. In this configuration, we also observe a decreased singlet return probability that is independent of the applied magnetic field and $\varepsilon$. We associate this decrease with the mixing between $|S\rangle$ and the unpolarized triplet state $\left|T_{0}\right\rangle$, driven by the Overhauser field gradient between the left and the multielectron dot. Similar features for the $2 N-4$ and
$2 N-2$ occupation of the multielectron dot are presented in Fig. 4, and are reminiscent of analogues $S-T_{0}$ mixing in two-electron double dots with sufficiently small exchange coupling [66].

The observed leakage spectrum in Fig. 3(c) can be reproduced qualitatively from our Hubbard model, even though it does not take into account Overhauser fields. First, we calculate the energy spectrum of the Hamiltonian of Eq. (2) using the same parameters as above, but at varying magnetic fields. We then identify the ground state associated with preparation of the double dot in the singlet state, and plot those values of $\varepsilon^{*}$ and $B_{\|}$for which this state crosses the fully polarized state $\left|T_{+}\right\rangle$. (For $B_{\|}<0$, we plot the crossing of the ground state with $\left|T_{-}\right\rangle$.) The state crossing in our model indicates where spin mixing due to Overhauser gradients is expected [Fig. 3(d)], in qualitative agreement with experimental data [Fig. 3(c)].

To confirm the origin of the decreased singlet probability in the $(1,0,2 N+1)$ configuration, we perform a timeresolved measurement of the Overhauser field gradient [17,87-91] between the leftmost dot and the multielectron dot. For that purpose, we fix the interaction point in the $(1,0,2 N+1)$ charge configuration and cyclically vary the waiting time $\tau$ from 0 to $100 \mathrm{~ns}$. The cycle is repeated continuously, keeping track of both the waiting time $(\tau)$ and laboratory time stamp $(\mathcal{T})$ associated with each single-shot readout. When plotting all individual single-shot readouts (singlet or triplet) versus their associated $\tau$ and $\mathcal{T}$ values [Fig. 3(e)], coherent oscillations between the singlet $|S\rangle$ and triplet $\left|T_{0}\right\rangle$ state become apparent within each column, with an oscillation frequency that slowly changes from column to column. This fluctuating behavior (shown here over a 30-slong laboratory time interval) is the hallmark of two-electron spin coherence interacting with the (diffusive) dynamics of the GaAs nuclear spin bath, and has been characterized in detail for two-electron double dots $[67,87,90,91]$. The high visibility of oscillations in Fig. 3(e)-here with frequencies fluctuating around approximately $50 \mathrm{MHz}$-confirms that
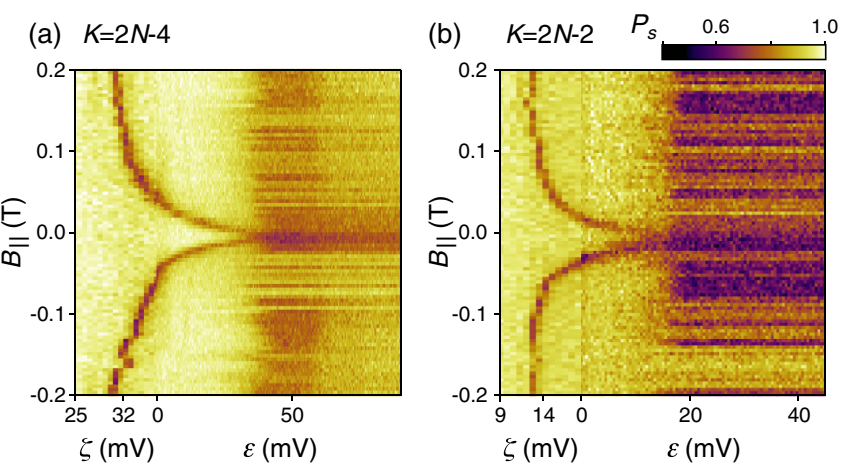

FIG. 4. Leakage spectroscopy of the double dot coupled to the multielectron dot at the transition between (a) $(2,0,2 \mathrm{~N}-2)$, $(1,1,2 N-2)$, and $(1,0,2 N-1)$ and (b) $(2,0,2 N-4)$, $(1,1,2 N-4)$, and $(1,0,2 N-3)$. 
the typical splitting between $|\uparrow \downarrow\rangle$ and $|\downarrow \uparrow\rangle$ states due to uncontrolled gradients of the Overhauser field-here approximately $0.2 \mu \mathrm{eV}$-dominates over the residual superexchange interactions in this regime. A more stringent bound for the strength of superexchange interactions between the unpaired spins in the $(1,0,2 N+1)$ configuration can be obtained from the Hubbard model: by evaluating the energy splitting in the vicinity of the transition to the $(1,1,2 N)$ configuration, we obtain $0.09 \mu \mathrm{eV}$ as an upper bound. The strength of superexchange can be enhanced by increasing the tunnel couplings [54], or by replacing the empty central dot by a multielectron quantum dot $[52,53,55]$.

\section{SPIN-1/2 BEHAVIOR FOR ODD OCCUPANCIES}

For odd occupation number the multielectron ground state must be spinful (all cases we study are consistent with spin-1/2), and accordingly, the resulting coupled spin system is more complex compared to the spinless case discussed in Sec. IV. To put our double-dot spin probe technique into context of previous experiments, we begin this section by reviewing a triple quantum dot in the threeelectron regime, before we turn to multielectron effects. The three-electron regime $(1,1,1)$ is difficult to realize in the geometry shown in Fig. 1(a). Therefore, on the same chip we activate another triple dot in which the lithographic size of the right dot is the same as the left and middle oneelectron dot, and present measurements for the $(1,1,1)$ regime of that device.

After describing the relevant physics of this tunnelcoupled $(1,1,1)$ system, using control parameters $\zeta$ and $\varepsilon$ as introduced in Fig. 1(c), we present multielectron effects for the $(1,1, K)$ system, where $K$ is large and odd. From measurements associated with each individual choice of $K$ alone, we find it difficult to distinguish a spin- $1 / 2$ ground state from spin-3/2 or higher, due to the similarity between the expected behaviors. However, in the context of the observed behavior of the next higher or lower even occupation, we find that assigning spin- $1 / 2$ to the ground state of all odd-occupied multielectron cases gives full consistency between the interpretations of all occupancies [92]. Therefore, in our analyses below we assume spin- $1 / 2$ ground states for all odd-occupied multielectron quantum dots. This choice is consistent with theoretical models for large two-dimensional quantum dots in GaAs, which for a realistic range of occupation numbers $(K=80-200)$ and interaction strength parameters $\left(r_{S}=1.4-2.5\right.$, based on experimental results from Refs. $[76,93,94])$ predict that the probability to observe spin-3/2 ground states is significantly reduced relative to the occurrence of spin- $1 / 2$ ground states [75]. Our measurements involve several odd occupancies of the multielectron dot and reveal that associated exchange profiles fall into characteristically distinct categories, which we reproduce and discuss further within a Hubbard model (see Sec. V E).

\section{A. Review: Three-electron triple quantum $\operatorname{dot}(K=1)$}

The $(1,1,1)$ charge state of a triple quantum dot, schematically illustrated in Fig. 5(a), allows for $2^{3}$ distinct spin states. The energy of these 8 three-electron states at finite external magnetic field are shown in Fig. 5(b). For $\varepsilon^{*}=0$, all degeneracies are removed by a combination of linear Zeeman coupling (independent of $\varepsilon^{*}$ ) and finite interdot tunneling (charge hybridization, $\varepsilon^{*}$ dependent). In particular, the state plotted in blue transforms (smoothly due to interdot tunneling) into a $(2,0,1)$ charge state when reducing $\varepsilon^{*}$. Accordingly, the spin state in this limit becomes equivalent to a spin singlet in the left dot, and a spectator spin in the right dot, represented as $|S ; \uparrow\rangle$. Unlike this "singletlike state," the state marked in red displays "tripletlike" behavior [95]: due to Pauli blockade it retains its $(1,1,1)$ charge character when reducing $\varepsilon^{*}$, and smoothly turns into a $T_{0}$-like state in the left double well and a spectator spin in the rightmost dot, represented as $\left|T_{0} ; \uparrow\right\rangle$. Many features of the spectrum in Fig. 5(b) have been studied previously in the context of exchange-only triple-dot spin qubits $[3,20,96,97]$, resonant exchange qubits $[19,98]$, and theoretical work on triple-dot spin

(a)

(b)
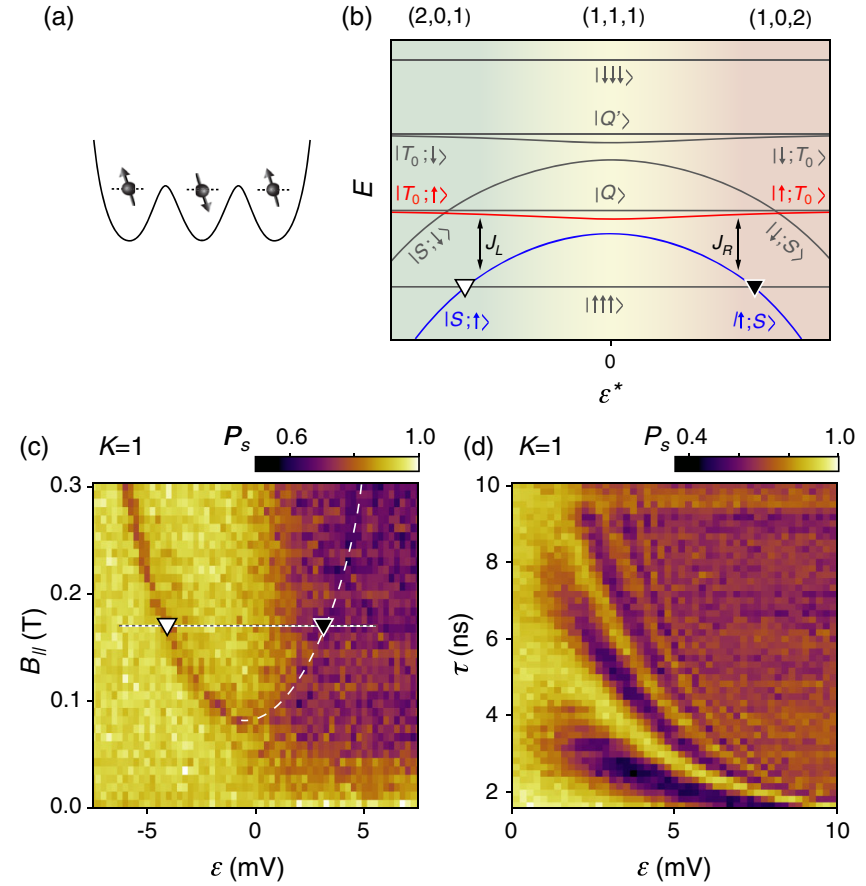

FIG. 5. (a) Schematic of the three-electron triple dot, which serves as a reference for discussing the odd-occupied multielectron dot tunnel coupled to the double dot. (b) Energy diagram of the three-electron triple-dot spin states for a finite external magnetic field. (c) Measured leakage spectrum for the threeelectron triple dot. Markers indicate leakage features attributed to the level crossings marked in (b). (d) Measured exchange oscillations reveal a monotonically increasing frequency, corresponding to monotonically increasing $J_{R}(\varepsilon)$ in (b). 
qubits [34,35,39,48,49,99-102] (for a review, see Ref. [103]).

Of interest here is the exchange energy between the singletlike and tripletlike state. In the vicinity of the $(2,0,1)$ $(1,1,1)$ charge transition we label this energy $J_{L}$, to indicate that it predominantly arises from tunneling across the left barrier (tunneling across the right barrier is suppressed, as the right dot is in deep Coulomb blockade at this detuning). Conversely, in the vicinity of the $(1,1,1)-(1,0,2)$ charge transition, exchange processes across the left barrier are negligible, while $J_{R}>0$ is significant. Accordingly, each eigenstate is labeled by its approximate spin texture, which in this region is a tensor product of the spin in the left dot, $|\uparrow\rangle$ or $|\downarrow\rangle$, and the two-electron spin state of the right double quantum dot, $|S\rangle$ or $\left|T_{i}\right\rangle$ (where $i=0,+,-$ ).

The splitting between the singletlike state and the tripletlike state [colored, respectively, blue and red in Fig. 5(b)], with respect to the difference $\varepsilon$ of gate voltages controlling occupancy of the left and right quantum dot, can be mapped out using leakage spectroscopy [97] [Fig. 5(c)] with a procedure similar to the one employed in Sec. IV. In this case, the system is prepared in the $|S ; \uparrow\rangle$ state, and the sharp feature of reduced $P_{S}$ indicates leakage from this singletlike state to the fully polarized $|\uparrow \uparrow \uparrow\rangle$ state [white and black triangles in Figs. 5(b) and 5(c)]. We observe that this feature diverges to high magnetic field for large positive and negative values of $\varepsilon$, consistent with the decrease of the energy of the singletlike state $|S ; \uparrow\rangle$ or $|\uparrow ; S\rangle$ in the $(2,0,1)$ or $(1,0,2)$ electron configuration, respectively.

As a side note, we mention that four of the $2^{3}$ triple-dot spin states form an $S=3 / 2$ quadruplet. An external magnetic field splits these according to spin projections $S_{z}= \pm 3 / 2$, labeled as $|\uparrow \uparrow \uparrow\rangle$ and $|\downarrow \downarrow \downarrow\rangle$, and $S_{z}= \pm 1 / 2$, labeled as $Q$ and $Q^{\prime}$.

The background of the leakage spectrum in Fig. 5(c) also shows an overall drop of $P_{S}$ with increasing $\varepsilon$, independent of the applied magnetic field. This indicates that the eigenstates on the left side $(\varepsilon<0)$ differ from the right side $(\varepsilon>0)$ of the spectrum. Once again, insight can be gained by reducing the interaction time $\tau$, which for $\varepsilon>0$ reveals coherent exchange oscillations between the middle spin and the right dot [Fig. 5(d)]. The frequency of the oscillations increases for larger values of $\varepsilon$, quantifying the increasing exchange coupling $J_{R}$ between the middle and the right quantum dot. This precession was previously exploited for the operation of the exchange-only qubit $[10,20,96]$. For this article, it serves the purpose of exemplifying that the spin of the right dot can be probed coherently using a proximal two-electron double dot.

This concludes our review of the three-electron triple dot. In the following sections we extend the same experimental concepts, namely leakage spectroscopy and measurement of exchange oscillations, to the system consisting of the two-electron double quantum dot coupled to the multielectron dot with an odd-occupancy spin-1/2 ground state. The role of $J_{L}$ and $J_{R}$ is played, respectively, by exchange coupling within the double dot $J_{\mathrm{DD}}$ and exchange coupling between the middle spin and the multielectron dot $J$ [compare Figs. 5(b) and 7(b) discussed below].

\section{B. Negative exchange interaction at the charge transition $(K=2 N-3,2 N-1)$}

We now focus on two particular odd occupancies of the multielectron quantum dot, $2 N-3$ and $2 N-1$, that turn out to behave similarly to each other but strikingly different than the $(1,1,1)$ system considered in Sec. VA. In these two cases the multielectron quantum dot has a single unpaired spin on the highest occupied orbital, while remaining electrons are paired up on lower-lying orbitals as spin singlets (see schematic in Fig. 6). The data we describe below [Figs. 7(c), 7(d), and 8] are interpreted within the Hubbard model associated with Fig. 6.

We first discuss leakage spectroscopy measurements for the multielectron quantum dot with $2 N-3$ occupancy. The left-hand part of Fig. 7(c) corresponds to a configuration in which the multielectron quantum dot is not significantly exchange coupled to the double quantum dot (i.e., $J \approx 0$ ). As expected from a conventional two-electron double dot, we observe in this regime a sharp feature of suppressed $P_{S}$ with a shape similar to the "spin funnel" presented in the left half of Fig. 3(c). Assuming that the multielectron dot simply constitutes a spectator spin in this regime (which can be representated as $|\uparrow\rangle$ anticipating the following analysis), we can associate this feature with the crossing

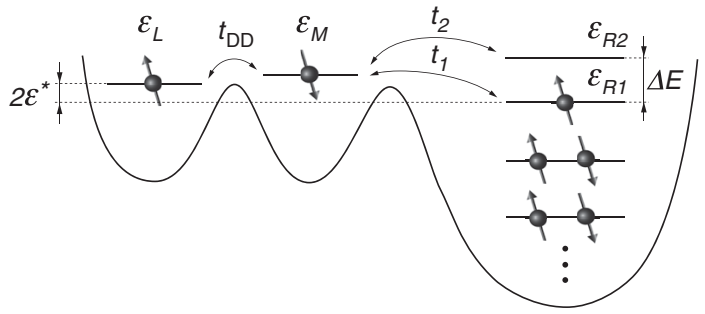

FIG. 6. Schematic of a two-electron double quantum dot coupled to an odd-occupied spin-1/2 multielectron dot. Symbols $\varepsilon_{L / M / R 1 / R 2}$ indicate single-particle energies of the lowest orbitals in the double dot and the two lowest orbitals above the effective vacuum in the multielectron dot. Arrows indicate tunnel couplings between the left and middle orbital $\left(t_{\mathrm{DD}}\right)$ and between the middle orbital and each of the two orbitals in the multielectron $\operatorname{dot}\left(t_{1 / 2}\right)$. The single-particle energy difference between the two orbitals on the multielectron dot is indicated by $\Delta E=\varepsilon_{R 2}-\varepsilon_{R 1}$. Detuning $\varepsilon^{*}=\left(\varepsilon_{L}-\varepsilon_{R 1}\right) / 2$ is varied when calculating the energy diagram and leakage spectrum presented in Figs. 7(b), 7(e), 9(b), and 9(e). Within the range of relevant parameters, lowlying orbitals in the right dot remain doubly occupied (i.e., spinless) and can be ignored when solving Eq. (3), thereby yielding a three-electron Hubbard model. [Similarly, a fourelectron Hubbard model is solved to model the spin-1 case in Figs. 13(b) and 13(e)]. 
(a)

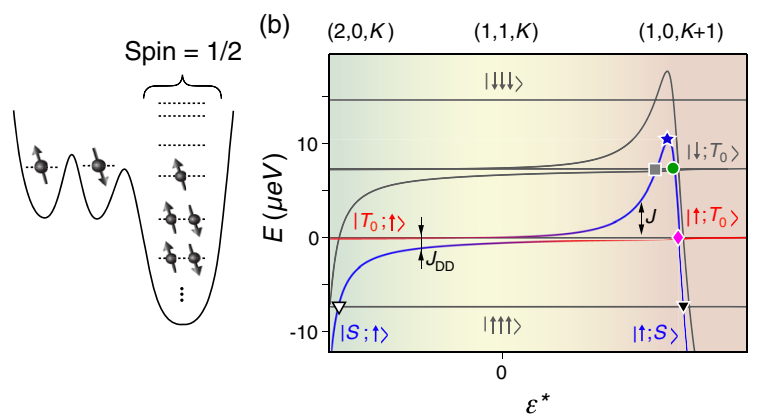

(c)

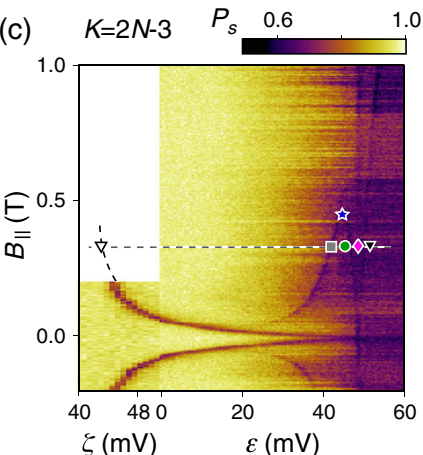

(e)



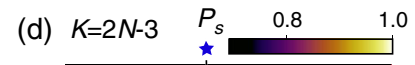
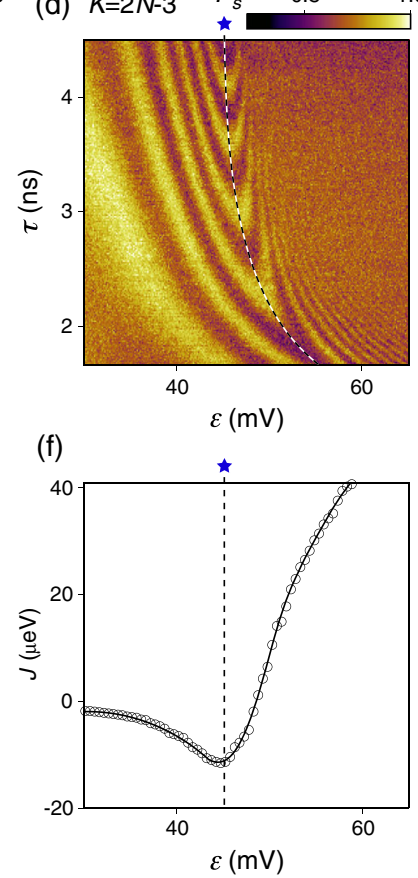

FIG. 7. (a) Schematic of the odd-occupied multielectron quantum dot with spin- $1 / 2$ ground state, tunnel coupled to the twoelectron double quantum dot. (b) The inferred energy diagram at the transition between $(2,0,2 N-3),(1,1,2 N-3)$, and $(1,0,2 N-2)$ charge configurations, for a finite magnetic field $B_{\|}=330 \mathrm{mT}$. The markers indicate crossings revealed by the leakage spectroscopy measurement presented in (c). Energies are measured relative to the energy of the state $|Q\rangle \propto|\uparrow \uparrow \downarrow\rangle+$ $|\uparrow \downarrow \uparrow\rangle+|\downarrow \uparrow \uparrow\rangle$. (d) Time-resolved measurement of exchange oscillations between the $2 N-3$ occupied spin-1/2 multielectron dot and the middle electron, obtained at zero magnetic field. The dashed line indicates pulse parameters for which the resulting quantum state is to first order insensitive to fluctuations in $\varepsilon$. (e) Leakage spectrum expected from the Hubbard model. (f) Exchange profile $J(\varepsilon)$ extracted from the pattern of exchange oscillations in (d).

between states $\mid S$; $\uparrow\rangle$ and $|\uparrow \uparrow ; \uparrow\rangle(=|\uparrow \uparrow \uparrow\rangle)$. In this interpretation, the curvature of the spin funnel (marked by a white triangle) reflects the gradual transition of the associated charge configuration from $(2,0,2 N-3)$ to $(1,1,2 N-3)$ occupancy.

For intermediate values of $\varepsilon$ the multielectron spin results in a leakage pattern that differs from the leakage spectrum

of a conventional three-electron triple quantum dot (discussed in Sec. VA). Namely, the line associated with the crossing between $|S ; \uparrow\rangle$ and $|\uparrow \uparrow \uparrow\rangle$ [white triangle in Fig. 7(c)] converges towards $B_{\|}=0$. Meanwhile, a second sharp feature emerges. With increasing $\varepsilon$, it first shifts towards larger values of $B_{\|}$(gray square), then reaches a maximum (blue star) before returning towards $B_{\|}=0$ (green circle). At the point where this feature crosses $B_{\|}=0$, we observe two additional sharp leakage features. The position of one of them is approximately independent of $B_{\|}$(pink diamond) while the other feature diverges towards large $B_{\|}$for increasing values of $\varepsilon$ (black triangle). This nontrivial leakage spectrum occurs at a detuning $(\varepsilon \gtrsim 30 \mathrm{mV}$ ) where the charge state of the ground state transitions into $(1,0,2 N-2)$.

To explain this peculiar leakage pattern, we modify the Hubbard model of Eq. (2) that successfully described the even-occupancy spin-0 case. Specifically, we now assume that the gate voltage of the multielectron quantum dot has been tuned such that the ground state is odd occupied, and is effectively described by a single unpaired spin on orbital $R 1$ (illustrated in Fig. 6). Lower-lying orbitals are assumed to be occupied by spinless electron pairs and are ignored. However, we found it necessary to include a higher-lying empty orbital $R 2, \Delta E$ higher compared to $R 1$. Therefore, the operator counting the number of relevant electrons in the right dot is now $\hat{n}_{R}=\hat{n}_{R 1}+\hat{n}_{R 2}$.

Including the spin correlation term $\xi$ of Eq. (1), this generalizes Eq. (2) to the following Hamiltonian, appropriate for the system illustrated in Fig. 6:

$$
\begin{aligned}
\hat{H}_{\text {spin- } 1 / 2}= & \sum_{i=L, M, R 1, R 2}\left(\varepsilon_{i} \hat{n}_{i}+\frac{U_{i}}{2} \hat{n}_{i}\left(\hat{n}_{i}-1\right)\right)+\sum_{i \neq j} \frac{K_{i j}}{2} \hat{n}_{i} \hat{n}_{j} \\
& -\frac{\xi}{2} \hat{S}^{2}+\frac{g \mu_{B} B_{\|}}{2}\left(\hat{n}_{\uparrow}-\hat{n}_{\downarrow}\right) \\
& -t_{\mathrm{DD}} \sum_{\alpha=\uparrow, \downarrow}\left(\hat{c}_{L, \alpha}^{\dagger} \hat{c}_{M, \alpha}+\hat{c}_{M, \alpha}^{\dagger} \hat{c}_{L, \alpha}\right) \\
& -t_{1} \sum_{\alpha=\uparrow, \downarrow}\left(\hat{c}_{M, \alpha}^{\dagger} \hat{c}_{R 1, \alpha}+\hat{c}_{R 1, \alpha}^{\dagger} \hat{c}_{M, \alpha}\right) \\
& -t_{2} \sum_{\alpha=\uparrow, \downarrow}\left(\hat{c}_{M, \alpha}^{\dagger} \hat{c}_{R 2, \alpha}+\hat{c}_{R 2, \alpha}^{\dagger} \hat{c}_{M, \alpha}\right) .
\end{aligned}
$$

The first line of this equation captures the gate-tunable chemical potentials and the Coulomb interactions, and hence, it is diagonal in terms of the spin occupancy numbers. As shown in Table II, we assume that the Coulomb interactions within a single orbital and between two orbitals of the multielectron quantum dot are equal. In the second line, the term proportional to $\xi$ captures the spin correlation energy: it is a phenomenological term that favors a $S=1$ triplet configuration when both levels $R 1$ and $R 2$ are occupied. Here, the operator of the multielectron quantum dot spin in orientation $j=x, y, z$ is 
TABLE II. Summary of parameters used in the Hubbard model for all presented simulations.

\begin{tabular}{lc}
\hline \hline Fixed parameters & Value (meV) \\
\hline$U_{L}=U_{M}$ & 5 \\
$U_{R}=U_{R 1}=U_{R 2}=K_{R 1, R 2}$ & 1 \\
$K_{L M}=K_{M R}=K_{M R 1}=K_{M R 2}$ & 0.1 \\
$K_{L R}=K_{L R 1}=K_{L R 2}$ & 0.02 \\
$\xi$ & 0.1 \\
$\varepsilon_{M}$ & 2 \\
$\bar{\varepsilon}$ & 0 \\
\hline \hline
\end{tabular}

$\hat{S}^{j}=\frac{1}{2} \sum_{\lambda, \alpha, \alpha^{\prime}} \hat{c}_{\lambda, \alpha}^{\dagger} \sigma_{\alpha, \alpha^{\prime}}^{j} \hat{c}_{\lambda, \alpha^{\prime}}$, where $\lambda=R 1, R 2$. This term is important when the single-particle spacing $\Delta E \equiv \varepsilon_{R 2}-\varepsilon_{R 1}$ is relatively small. The term proportional to $B_{\|}$describes the Zeeman splitting. The remaining terms proportional to $t_{\mathrm{DD}}, t_{1}$, and $t_{2}$ are chosen to be real and positive. They describe, respectively, tunnel couplings within the double quantum dot, between the middle $\operatorname{dot} M$ and $R 1$, and between $M$ and $R 2$.

In Fig. 7(b), we present the energy diagram of the double quantum dot coupled to the spin- $1 / 2$ multielectron quantum dot, calculated from the three-electron spectrum of Eq. (3) using $t_{1}=12 \mu \mathrm{eV}, t_{2}=48 \mu \mathrm{eV}, t_{\mathrm{DD}}=12 \mu \mathrm{eV}$, and $\Delta E=160 \mu \mathrm{eV}$. All plotted energies are measured relative to the energy of the state $|Q\rangle \propto|\uparrow \uparrow \downarrow\rangle+|\uparrow \downarrow \uparrow\rangle+|\downarrow \uparrow \uparrow\rangle$, which appears at $E=0$. In such a plot, tripletlike states display constant energies, whereas singletlike states depend on detuning.

Again, we have inspected two relevant states (marked blue for a singletlike state and red for a tripletlike state) in more detail, and indicate their spin states in the limit of very negative and very positive detunings. Further, for negative $\varepsilon^{*}$, we have labeled their energy splitting by $J_{\mathrm{DD}}$, to indicate that this exchange coupling arises predominantly from interdot tunneling within the double dot (discussed in the next paragraph). For positive $\varepsilon^{*}$, interdot tunneling within the double dot is negligible, but tunneling between the middle dot and the multielectron dot is important. The resulting exchange coupling, labeled $J$, reflects nontrivial spin-correlation effects arising from the orbitals within the multielectron dot (discussed below).

The left-hand part of Fig. 7(b), characterized by finite $J_{\mathrm{DD}}$ and negligible $J$, has an interpretation very similar to the left-hand part of Fig. 5(b), i.e., a three-electron triple dot with finite $J_{L}$ and negligible $J_{R}$. In particular, the crossing between $|S ; \uparrow\rangle$ and $|\uparrow \uparrow \uparrow\rangle$ states (white triangle) is expected to result in a spin-funnel-like feature in this regime, for the same reasons as in Sec. VA.

For increasing values of $\varepsilon^{*}$, however, the singletlike state $|S ; \uparrow\rangle$ continuously changes its spin texture from $|\uparrow \downarrow \uparrow\rangle-$ $|\downarrow \uparrow \uparrow\rangle$ to $|\uparrow \downarrow \uparrow\rangle+|\uparrow \uparrow \downarrow\rangle$, becoming a tripletlike state $\left|\uparrow ; T_{0}\right\rangle$ (we omit normalization). Concurrently, the tripletlike state $\left|T_{0} ; \uparrow\right\rangle$ continuously changes its spin texture from $|\uparrow \downarrow \uparrow\rangle+$ $|\downarrow \uparrow \uparrow\rangle$ to $|\uparrow \downarrow \uparrow\rangle-|\uparrow \uparrow \downarrow\rangle$, becoming a singletlike state $|\uparrow ; S\rangle$ for large $\varepsilon^{*}$. In the model, this transition is driven by the negative exchange interaction arising from $\xi=0.1 \mathrm{meV}$ in conjunction with large tunneling $t_{2}$ to the second orbital [cf. Eq. (3)], which increases the energy of the singletlike state $|\uparrow ; S\rangle$ relative to the tripletlike state $\left|\uparrow ; T_{0}\right\rangle$. However, for even larger $\varepsilon^{*}$ [i.e., in the $(1,0, K+1)$ charge configuration], the tunneling effects become suppressed, and hence, the singletlike state $|\uparrow ; S\rangle$ becomes the ground state due to a relatively large level spacing $\Delta E>\xi$.

The negative sign of the exchange interaction $J$ for intermediate values of $\varepsilon^{*}$ explains why for Zeeman splittings smaller than the maximum energy (blue star) two additional crossings are expected [marked in Fig. 7(b) by gray square and green circle], consistent with features observed in the leakage spectrum of Fig. 7(c). For both crossings we expect leakage from $|\uparrow ; S\rangle$ into $\left|\uparrow ; T_{-}\right\rangle$, as this state has total spin projection $S_{z}=-1 / 2$ and is accessible via electron-nuclear flip-flop processes.

In the context of these results from the Hubbard model [Fig. 7(b)], we are able to return to the measurements [Figs. 7(c), 7(d), and 8] and discuss a few more details.

For sufficiently large values of $\varepsilon^{*}$ in Fig. 7(b), the energy of the singletlike state $|\uparrow ; S\rangle$ decreases and becomes lower than the energy of the tripletlike state $\left|\uparrow ; T_{0}\right\rangle$. For the crossing of these two states we expect a leakage feature (indicated by the pink diamond), at a detuning value that is independent of the magnetic field (since the two involved states have the same spin projection). For higher detuning the energy of the $|\uparrow ; S\rangle$ state further decreases, and crosses the $|\uparrow \uparrow \uparrow\rangle$ state, resulting in the leakage feature indicated by a black triangle. Indeed, both leakage features are clearly observed in the experiment, as indicated by the pink diamond and black triangle in Fig. 7(c). In particular, the divergence of one leakage feature for increasing $\varepsilon$ (black triangle) implies that the multielectron quantum dot in $2 N-2$ occupancy has a spin- 0 ground state, consistent with the evidence presented in Sec. IV.

The measured leakage spectrum does not reveal the crossing between $|\uparrow ; S\rangle$ and the fully polarized $|\downarrow \downarrow \downarrow\rangle$ state. This is expected, as leakage into the $|\downarrow \downarrow \downarrow\rangle$ state
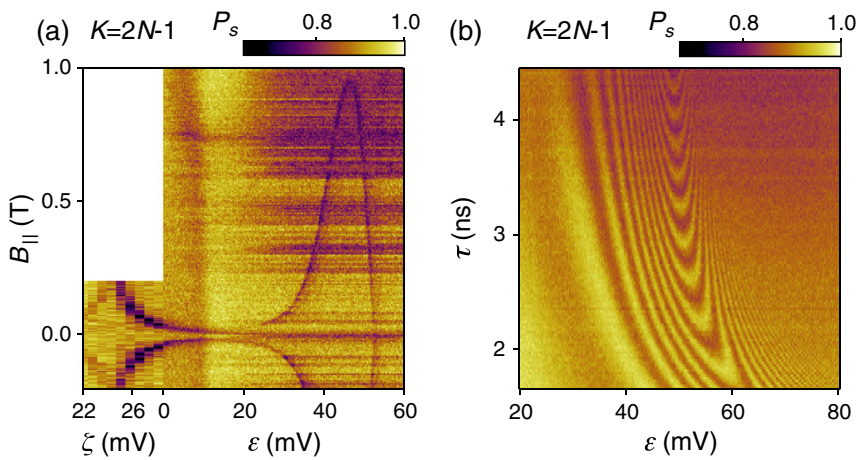

FIG. 8. Leakage spectroscopy (a) and time-resolved measurement of exchange oscillations (b) for the multielectron dot occupied by $2 N-1$ electrons. 
$\left(S_{z}=-3 / 2\right)$ would require a change of the electronic spin projection by 2 (which is not expected for weak spin-orbit interaction and typical Overhauser field gradients).

An identical analysis of the leakage spectroscopy measurements for the $2 N-1$ occupancy of the multielectron quantum dot [Fig. 8(a)] yields the same conclusion. In particular, it indicates that the multielectron dot with $2 \mathrm{~N}$ occupancy has a spin- 0 ground state, in agreement with the evidence presented in Sec. IV.

Similar to our procedure in Sec. IV, we extract the relevant state crossings from calculations as in Fig. 7(b) for varying external magnetic fields, and thereby generate the leakage spectrum expected for the system of Fig. 6. The resulting leakage map, shown in Fig. 7(e), qualitatively reproduces all features of the leakage spectroscopy measurements. This calculation also predicts leakage between the tripletlike state $\left|T_{0} ; \uparrow\right\rangle$ and a state with $S=+3 / 2$, $S_{z}=+3 / 2$, which we plot in gray color. This leakage feature is not apparent in the measured data, as our initialization pulses were designed to prepare the $|S ; \uparrow\rangle$ state, which is orthogonal to the states that anticross.

The interpretation of the maximum in Fig. 7(c) (blue star) as an extremum in $J(\varepsilon)$ can be confirmed directly in the time domain, by reducing the interaction time $\tau$ and inspecting coherent oscillations between the singletlike and tripletlike state at intermediate values of $\varepsilon$. In this technique, the oscillation frequency observed at detuning $\varepsilon$ is a quantitative measure for $|J(\varepsilon)|$ [96]. The experimental data for $2 N-3$ and $2 N-1$ occupancy of the multielectron dot are presented in Figs. 7(d) and 8(b), respectively. In both cases, an increase followed by a decrease in oscillation frequency with increasing detuning is clearly observed. For comparison, the exchange energy extracted from the leakage spectroscopy pattern for $2 N-3$ occupancy is presented in Fig. 7(f). We observe that the minimum of $J(\varepsilon)$ in Fig. 7(f) occurs at that value of $\varepsilon$ for which the oscillation frequency in Fig. 7(d) shows a maximum (for large $\tau$ this agreement is good, whereas for small $\tau$ pulse distortions arising from finite-rise-time effects associated with our cryostat wiring become significant). The overall agreement between maxima in leakage spectra [blue star in Figs. 7(c) and 8(a)] and maxima in oscillation speed [Figs. 7(d) and 8(b)] confirms that the exchange interaction strength has an extremum as a function of $\varepsilon$. Although the oscillations in Figs. 7(d) and 8(b) do not reveal the absolute sign of the exchange coupling, these measurements do confirm its change of sign [61].

The presence of an extremum in the exchange profile $J(\varepsilon)$, indicated by a blue star in Fig. 7, implies that the exchange splitting is to first order insensitive to detuning fluctuations. For low-frequency $\varepsilon$ noise, symmetric operating points that take the finite experimental pulse rise time into account, indicated with a dashed line in Fig. 7(d), can then be exploited to perform high-fidelity exchange gates $[25,26]$. Although the residual sensitivity to fluctuating tunnel barriers remains to be investigated, coherent spin exchange processes demonstrated across a multielectron dot already showed an improvement at such noise-robust operating points [55].

To summarize, the qualitative agreement between observed and expected features leads us to accept the physical inspection of the Hubbard model results [Fig. 7(b)] as the correct interpretation of the measurement results [Figs. 7(c), 7(d), and 8]. This allows us to conclude that for intermediate values of $\varepsilon$, the tripletlike configuration associated with $\left|\uparrow ; T_{0}\right\rangle$ has a lower energy than the singletlike state associated with $|\uparrow ; S\rangle$. We refer to this inversion as negative exchange coupling. For both the $2 N-3$ and $2 N-1$ occupancy of the multielectron quantum dot we observe this negative (i.e., triplet-preferring) exchange coupling to the proximal electron spin, as long as the proximal spin resides in the middle dot, i.e., for charge configurations $(1,1,2 N-3)$ or $(1,1,2 N-1)$. Once a sufficiently large detuning voltage transfers the proximal electron onto the multielectron dot, i.e., resulting in a $(1,0,2 N-2)$ or $(1,0,2 N)$ charge configuration, the exchange interaction becomes positive, consistent with a spin-0 ground state for the even-occupied multielectron quantum dot (studied in Sec. IV).

\section{Negative exchange within the multielectron $\operatorname{dot}(K=2 N+1)$}

Next, we focus on the $2 N+1$ occupation of the multielectron dot. Similarly to the odd occupancies $2 N-3$ and $2 N-1$ (Sec. V B), we expect that a single unpaired electron occupies the highest occupied orbital of the multielectron dot [Fig. 9(a)]. However, the leakage spectroscopy measurement [Fig. 9(c), discussed below] implies that the exchange interaction with the neighboring middle electron is qualitatively different. By changing the model parameters associated with Fig. 6 slightly (in particular, the relative magnitudes of $t_{1}, t_{2}, \Delta E$, and $\xi$ ), the calculated spectrum can be made to match the experimental data.

The left-hand side of Fig. 9(c) presents an experimental leakage spectrum similar to that observed for $2 N-3$ and $2 N-1$, and hence, we tentatively associate the sharp funnel-like leakage feature (white triangle) with the crossing between singletlike state $|S ; \uparrow\rangle$ and the fully polarized state $|\uparrow \uparrow \uparrow\rangle$. In contrast to the previous cases, this feature does not converge to $B=0$ for increasing $\varepsilon$, but increases to high magnetic fields (gray square) for intermediate values of $\varepsilon$. This increase occurs within the $(1,1,2 N+1)$ charge state, indicating that the singletlike state $|\uparrow ; S\rangle$ has a lower energy than the tripletlike state as long as the proximal electron resides on the middle dot. For larger detuning, around a charge transition to $(1,0,2 N+2)$ charge state, this leakage feature reaches a (sharp) maximum and then crosses through $B_{\|}=0$ (pink diamond), along with two sharp leakage features appearing. 
(a)
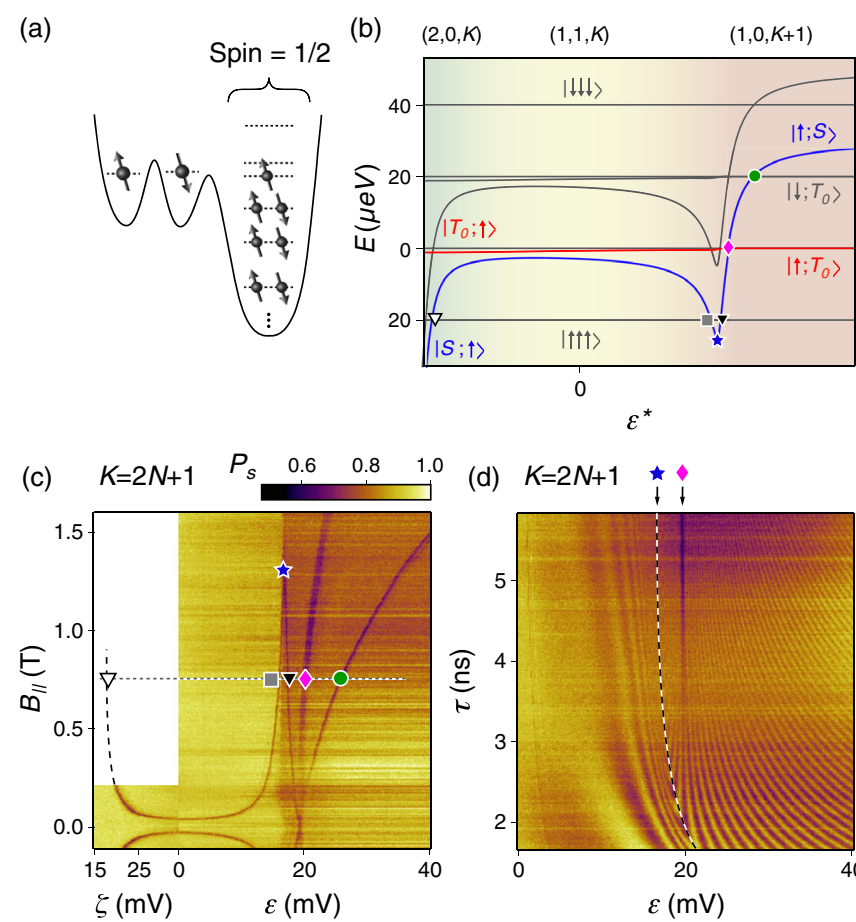

(e)

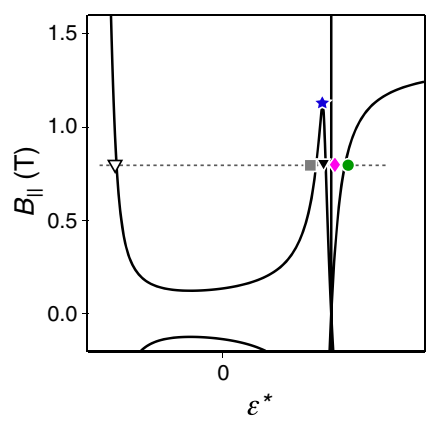

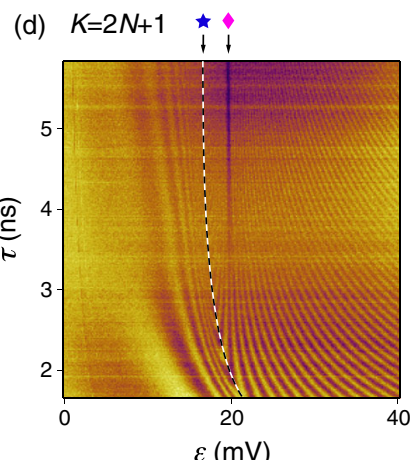

(f)

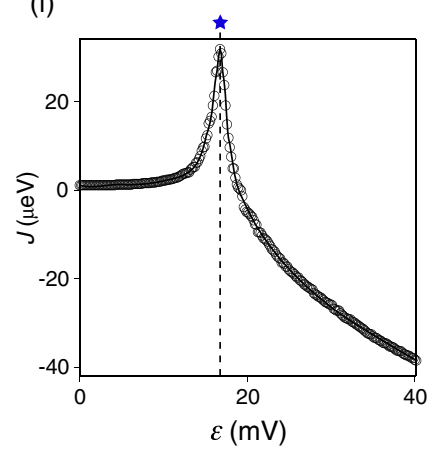

FIG. 9. (a) Schematic of the odd-occupied multielectron quantum dot with spin- $1 / 2$ ground state, tunnel coupled to the twoelectron double quantum dot. (b) Calculated energy diagram at the transition between $(2,0,2 N+1),(1,1,2 N+1)$, and $(1,0,2 N+2)$ charge configurations, for a finite magnetic field $B_{\|}=900 \mathrm{mT}$ and input parameters motivated by the observed spectrum in (c). Markers indicate crossings revealed by the leakage spectroscopy measurement presented in (c). Energies are measured relative to the energy of the state $|Q\rangle \propto|\uparrow \uparrow \downarrow\rangle+$ $|\uparrow \downarrow \uparrow\rangle+|\downarrow \uparrow \uparrow\rangle$. (d) Time-resolved measurement of exchange oscillations between the $2 N+1$ occupied spin- $1 / 2$ multielectron quantum dot and the middle electron, obtained at zero magnetic field. Color scale as in (c). The dashed line indicates pulse parameters for which the resulting quantum state is to first order insensitive to fluctuations in $\varepsilon$. (e) Leakage spectrum expected from the Hubbard model. (f) Exchange profile $J(\varepsilon)$ extracted from the pattern of exchange oscillations in (d).

This leakage spectrum can be reproduced within our model (Fig. 6) using $t_{1}=30 \mu \mathrm{eV}, t_{2}=6 \mu \mathrm{eV}, t_{\mathrm{DD}}=$ $20 \mu \mathrm{eV}$, and $\Delta E=30 \mu \mathrm{eV}$. The calculated energy diagram, obtained from the Hamiltonian Eq. (3), is presented

in Fig. 9(b). For these parameters, the spectrum reveals a positive (singlet-preferring) exchange interaction for the $(1,1,2 N+1)$ configuration and a negative (tripletpreferring) exchange interaction for $(1,0,2 N+2)$. Moreover, we can associate all leakage features observed in Fig. 9(c) with specific crossings in the calculated spectrum. In particular, the three sharp leakage features converging towards $B_{\|}=0$ correspond to the crossings between $|\uparrow ; S\rangle$ and states with total spin $S=3 / 2$ and spin projection, respectively, $S_{z}=+3 / 2$ (black triangle), $+1 / 2$ (pink diamond), and $-1 / 2$ (green circle).

Following the reasoning from Sec. V B, we come to the conclusion that the $2 N+2$ occupied multielectron dot has a spin-1 ground state. Indeed, in Sec. VI we present exchange effects in the $(1,1,2 N+2)$ system that are consistent with a spin-1 ground state of the multielectron dot.

In Fig. 9(d), we present time-resolved exchange oscillations measured for the same configuration as for the leakage spectroscopy in Fig. 9(c). As for the $2 N-3$ and $2 N-1$ occupancies, we find that the oscillation frequency reaches a maximum for the same value of $\varepsilon$ as the local maximum in the leakage spectrum (blue star). [Deviations appear for short values of $\tau$, due to finite-rise-time effects as in Figs. 7(d) and 8(b).] For the highest values of $\tau$ we additionally observe a suppression of $P_{S}$ at $\varepsilon \approx 21 \mathrm{mV}$, which we attribute to the onset of incoherent leakage from the singletlike state into the $|\uparrow ; S\rangle$ state (pink diamond).

As in Sec. V B, we note the appearance of extrema in the exchange profile of Fig. 9, which possibly are useful for the execution of charge-noise-insensitive exchange gates. More details about the charge occupancy presented in this section $(K=2 N+1)$, including tunability of the exchange profile, can be found in Ref. [61].

\section{Other odd occupancies of the multielectron quantum dot $(K=2 N-5, K=2 N+3)$}

We now present the results of leakage spectroscopy and exchange-oscillation measurements for the $2 N-5$ and $2 N+3$ occupancies of the multielectron dot. These are the most extreme occupancies studied in this work. Further addition or removal of electrons is possible, but would require significant changes of several tuning voltages to maintain a useful tunnel coupling between the middle and multielectron dot, presumably due to considerable changes of the quantum dot area. The observed behavior is consistent with the ground states found for the other odd occupations, namely a spin- $1 / 2$ multielectron dot, but the reduced quality of data did not allow an analysis as detailed as that in Secs. V B and V C.

In Fig. 10(a), we present leakage spectroscopy for the $2 N-5$ occupancy of the multielectron quantum dot. We attribute the two funnel-like features in the left half of the panel with the usual exchange coupling within the twoelectron double quantum dot, decreasing in strength with increasing $\varepsilon$. However, at $\varepsilon \approx 20 \mathrm{mV}$ each feature appears 

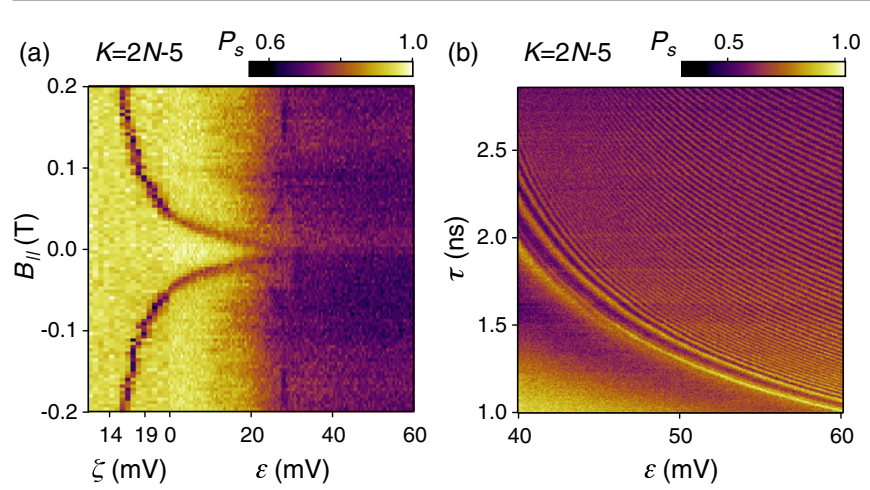

FIG. 10. Leakage spectroscopy (a) and time-resolved measurement of exchange oscillations (b) for the multielectron dot occupied by $2 N-5$ electrons.

to split into two features. One converges towards $B=0$ while the other quickly increases and possibly reaches a maximum at $\varepsilon \approx 28 \mathrm{mV}$ before returning and crossing $B=0$ at about $\varepsilon=30 \mathrm{mV}$. On the one hand, this may indicate that the exchange interaction strength between the middle electron and the spin- $1 / 2$ multielectron dot has a negative sign for small wave function overlap [i.e., in the $(1,1,2 N-5)$ charge configuration] and positive for large wave function overlap [i.e., in the $(1,0,2 N-4)$ charge configuration]. On the other hand, the opposite behavior (i.e., exchange sign going from positive to negative) would also be consistent with the leakage pattern, although we dismiss this possibility based on the spin- 0 behavior presented for $K=2 N-4$.

Exchange oscillations for $2 N-5$ occupancy are presented in Fig. 10(b). Notably, there is no indication for a local maximum in the oscillation frequency, in contrast to an extremum in the exchange interaction strength that we inferred from the leakage spectrum in Fig. 10(a). We do not understand the absence of an extremum in Fig. 10(b), but note that the presence of exchange oscillations by itself is evidence for a spinful ground state of the $2 N-5$ occupied multielectron dot.

Leakage spectroscopy performed for the $2 N+3$ occupation, presented in Fig. 11(a), reveals characteristics similar to those of the conventional three-electron triple quantum dot (Sec. VA). This similarity, and the absence of unusual leakage features at the $(1,1,2 N+3)$ to $(1,0,2 N+4)$ charge transition, suggests that the multielectron quantum dot with this occupancy behaves as an ordinary spin-1/2 dot. However, we cannot fully exclude the possibility that at high $\varepsilon$ the exchange interaction reaches a maximum and possibly changes sign, as such a behavior is hard to detect for large tunnel couplings [61]. In addition, the observed pattern of exchange oscillations in Fig. 11(b) is not quite clear enough to support the presence or absence of an extremum in the exchange profile, due to an increased dephasing rate at the interdot charge transition [104].
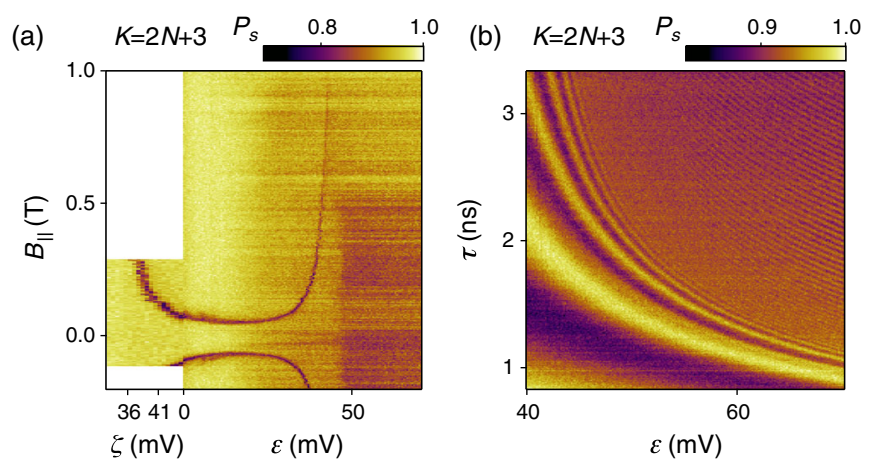

FIG. 11. Leakage spectroscopy (a) and time-resolved measurement of exchange oscillations (b) for the multielectron dot occupied by $2 N+3$ electrons.

\section{E. Different exchange profiles for a spin-1/2 multielectron dot expected from the Hubbard model}

According to our phenomenological model, the effective exchange coupling between a spin-1/2 ground state of the multielectron dot and the middle spin depends on the precise choice of the various input parameters. However, the general behavior of the exchange profile falls into four main regimes, as shown schematically in Fig. 12.

In regime I, the effective exchange coupling is always positive (singlet preferring) as $\varepsilon^{*}$ is tuned towards the charge transition from $(1,1, K)$ to $(1,0, K+1)$, where an additional electron moves onto the multielectron dot. The behavior is qualitatively similar to that of a three-electron triple dot $(K=1$, Sec. VA), and is possibly that observed for $K=2 N+3$ (Sec. V D).

In regime II, the effective exchange coupling is negative (triplet preferring) in the $(1,1, K)$ charge configuration but becomes positive as the charge transition to $(1,0, K+1)$ is approached. Consequently, the exchange profile includes a maximum and a zero crossing. This is the regime observed for $2 N-3$ and $2 N-1$ occupancies (Sec. V B).

Regime III is similar to regime I for low detuning in the $(1,1, K)$ charge configuration, with positive exchange, but the exchange reverses sign and becomes a negative exchange very close to the charge transition to the $(1,0, K+1)$ state, as observed for $2 N+1$ occupancy (Sec. V C).

Regime IV is characterized by a negative exchange coupling in the $(1,1, K)$ configuration close to $(1,0$, $K+1)$, and the exchange coupling remains negative for higher detuning. Note that there is no zero crossing in the exchange profile, even though the exchange coupling for low detuning (corresponding to exchange between middle dot and left dot) is positive, and exchange and high detuning (corresponding to exchange between middle dot and the multielectron dot) is negative. This is because in the charge configuration $(1,1, K)$, tunneling across the left and right barrier are both present, and hence, eigenstates cannot be decomposed into a product state of one spectator spin (in either left or right dot) and a remaining 


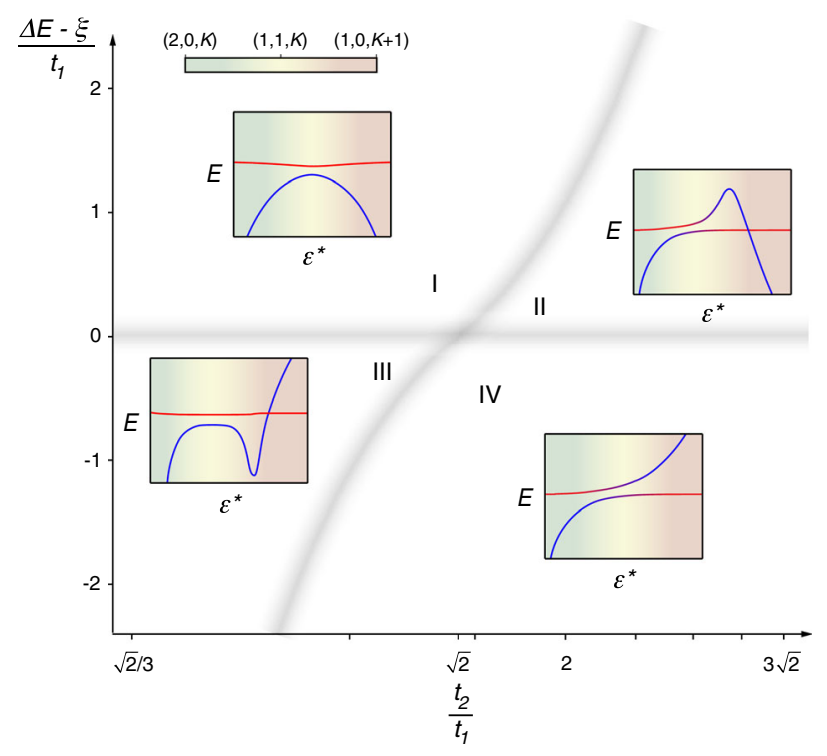

FIG. 12. Illustration of qualitatively different exchange profiles arising from the interplay between the level spacing in the multielectron quantum dot $\Delta E$, spin correlation energy $\xi$, and tunnel couplings between a single-electron dot and two lowest orbitals of the multielectron quantum dot $t_{1 / 2}$. Colored lines in the insets I-IV represent the energies of the three-spin states with $S=1 / 2, S_{z}=-1 / 2$ as a function of detuning $\varepsilon^{*}$.

spin-singlet (or spin triplet) state. In other words, the energy spitting between eigenstates is nonzero at this detuning, but cannot be classified as positive or negative because the eigenstates themselves are superpositions of singletlike (blue) and tripletlike (red) states. Although the measurements of $K=2 N-5$ share some properties of regime IV, this regime has not been clearly observed.

In Figure 12 we intentionally omit units on the four insets, to emphasize that our simple theoretical model predicts four qualitatively different regimes of exchange profiles. However, only regimes II and III have been observed unambiguously in our multielectron device (regime I has been observed only for $K=1$ ). To gain insight into the physics that-within the theoretical model - gives rise to these four regimes, we can inspect the crossover between these regimes in more detail. In particular, we can estimate the location of the boundaries between these four regimes by analyzing the role of two dimensionless quantities.

The first parameter is $(\Delta E-\xi) / t_{1}$, and constitutes the vertical axis of Fig. 12. This parameter can be positive or negative, depending on the relative strength of the spin correlations. When positive, i.e., $\Delta E>\xi$, the energy separation of the two relevant single-particle levels 1 and 2 in the multielectron dot is larger than the spin correlation energy, thereby suppressing the formation of a high-spin ground state. Accordingly, if the middle electron is transferred into the right dot (large detuning), its lowest energy state in the $(1,0, K+1)$ charge configuration will be a singlet configuration with level 1 doubly occupied. When this parameter is negative, i.e., $\Delta E<\xi$, the spin correlation energy is larger than the kinetic energy required to form a high-spin state, and hence, a triplet configuration with an electron in both level 1 and level 2 is energetically preferred in the $(1,0, K+1)$ charge configuration. In the limit $\Delta E \ll \xi$, and ignoring the low spatial symmetry of the mesoscopic multielectron dot, such a high-spin filling of the "shell" spanned by levels 1 and 2 can be viewed as the manifestation of Hund's rule in a 2DEG-based artificial atom $[72,77,105]$.

Near the charge transition from $(1,1, K)$ to $(1,0, K+1)$, the effective exchange coupling between the multielectron dot and the double quantum dot results from a competition between a positive (singlet-preferring) contribution and a negative (triplet-preferring) contribution. These contributions arise from virtual transitions to the two doubly occupied configurations of the multielectron dot. The relative size of these contributions depends on the tunnel couplings $t_{1}$ and $t_{2}$, and consequently, the second parameter we use to describe the spectrum is the ratio $t_{2} / t_{1}$. This parameter forms the horizontal axis of Fig. 12.

For large detuning, i.e., when the charge configuration of the ground state is $(1,0, K+1)$, the exchange splitting is simply the energy difference between the doubly occupied triplet and singlet states set by the Hubbard model: $\Delta E-\xi$. Therefore, the line $(\Delta E-\xi) / t_{1}=0$ represents the crossover from regimes I and II, which have singlet ground states at large detuning $(\Delta E-\xi>0)$, to regimes III and IV, which have triplet ground states at large detuning $(\Delta E-\xi<0)$.

At small detuning, i.e., when the charge configuration of the ground state is predominantly $(1,1, K)$, the Hubbard model can be analyzed perturbatively. In this regime the positive contribution to the exchange is approximately $J_{S}=2 t_{1}^{2} / \Delta E_{S}$, where $\Delta E_{S}$ is the difference in energy to the doubly occupied singlet state. $\Delta E_{S}$ changes with $\varepsilon^{*}$ and decreases as the system is tuned towards the charge transition. The negative contribution is approximately $J_{T}=-t_{2}^{2} / \Delta E_{T}$, where $\Delta E_{T}$ is the difference in energy to the doubly occupied triplet state. (Note that $\left.\Delta E_{T}-\Delta E_{S}=\Delta E-\xi\right)$.

We focus first on the region where $(\Delta E-\xi) / t_{1}>0$, so that $\Delta E_{S}<\Delta E_{T}$ and the system is in either regime I or II. Consider the exchange in the $(1,1, K)$ charge configuration not too far from the charge transition to $(1,0, K+1)$, so that the exchange is dominated by coupling to the multielectron dot and the effect of the left dot can be neglected. When $t_{2} / t_{1}>\sqrt{2}$ and $\Delta E-\xi$ is not too large, we expect that $J_{S} \lesssim\left|J_{T}\right|$, and so the ground state is a triplet and the effective exchange is negative. Consequently, the ground state must change from triplet to singlet as $\varepsilon^{*}$ is tuned through $(1,1, K)$ towards $(1,0, K+1)$. This places the system in regime II. For larger values of $t_{1}$ and smaller values of $t_{2}$, the ground state in $(1,1, K)$ is a singlet throughout the charge transition and the system is in regime 
I. An approximate location for the crossover between regime I and regime II is the locus of points where $J_{S}=$ $\left|J_{T}\right|$ when $\Delta E_{S} \simeq t_{1}$. This choice ensures that regime II is characterized by a triplet ground state until $\varepsilon^{*}$ is tuned close enough to the charge transition that the tunneling starts to strongly mix the two charge configurations and perturbative expressions for exchange are no longer valid. This boundary is indicated in Fig. 12, and we verified that spectra of the full Hubbard model are in agreement with this choice.

The boundary between regime III and regime IV sits in the region where $(\Delta E-\xi) / t_{1}<0$, and so $\Delta E_{T}<\Delta E_{S}$. Following the same reasoning as for the boundary between I and II, the crossover between regime III and regime IV lies near the locus of points where $J_{S}=\left|J_{T}\right|$ when $\Delta E_{T} \simeq t_{1}$. This boundary is likewise indicated in Fig. 12 .

As pointed out at the beginning of this section, regimes II and III have clearly been observed in our experiment. This may hint at electrons occupying orbitals at a higher energy (i.e., with larger kinetic energy) having an increased tendency to penetrate the potential barrier between the dots (i.e., resulting in $t_{2}>t_{1}$ ). The behavior for $K=1$ is clearly that of regime I, whereas the observed behaviors for $K=2 N+3$ and $K=2 N-5$ are less clear (possibly regime I and IV). Overall, the number of occupancies studied in this work is too small to draw any general conclusions. To resolve this question, it would be beneficial to investigate more devices or to use distorting gates [56] to change the quantum dot potential and thereby gather meaningful statistics.

\section{SPIN-1 BEHAVIOR FOR $K=2 N+2$}

Our final case concerns the $2 N+2$ occupancy of the multielectron quantum dot, which showed different characteristics than the other even occupancies presented. From the behavior of the $2 N+1$ occupancy (Sec. VC), we conclude that the addition of one electron to the spin-1/2 ground state results in a triplet configuration that has a lower energy than the singlet configuration. Our expectation, therefore, is that the $2 N+2$ ground state of the multielectron dot shows spin-1 behavior [Fig. 13(a)].

Indeed, the leakage spectroscopy data, featuring a prominent U-shaped leakage feature in Fig. 13(c), is more similar to that of a three-electron triple dot [Fig. 5(c)] than to that associated with other even occupations [Figs. 3(c) and 4]. This points towards the presence of a nonzero spin in the multielectron quantum dot, which we associate with spin-1 in this case. We note that the sharp leakage feature diverges to large $B_{\|}$for increasing $\varepsilon$, indicating a positive sign of the exchange interaction (i.e., preferring the lowspin state). Further, measurement of exchange oscillations clearly shows the presence of coherent exchange interactions [Fig. 13(d)]. These observations lead us to the following conclusions. First, the $2 N+2$ occupied multielectron dot carries a nonzero spin. Second, the exchange (a)
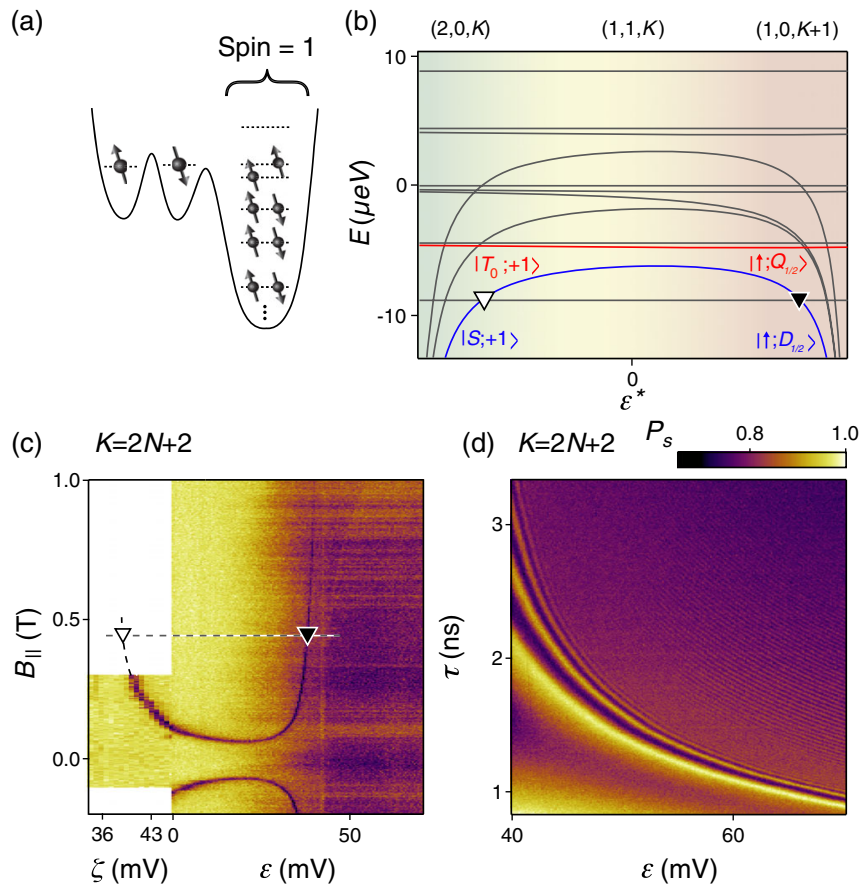

(e)

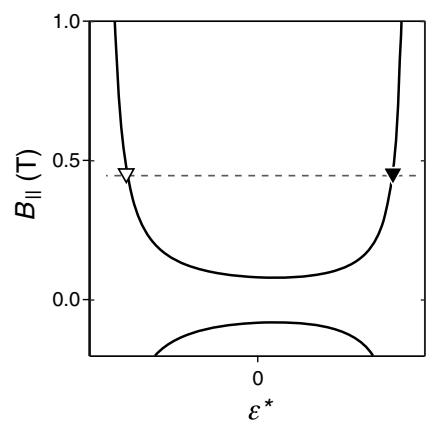

(f)
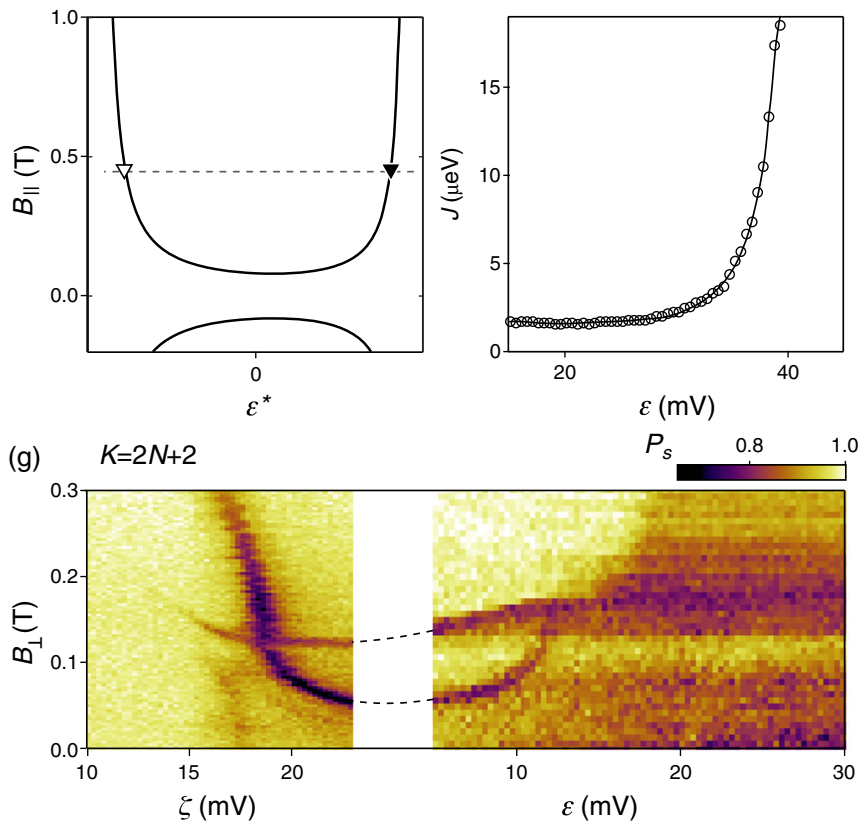

FIG. 13. (a) Schematic of the even-occupied multielectron quantum dot with spin-1 ground state, tunnel coupled to the twoelectron double quantum dot. (b) The inferred energy diagram at the transition between $(2,0,2 N+2),(1,1,2 N+2)$, and $(1,0,2 N+3)$ electronic configurations, for a finite magnetic field. The markers indicate the crossings revealed by leakage spectroscopy presented in (c). (d) Time-resolved measurement of exchange oscillations between the $2 N+2$ occupied spin- $1 / 2$ multielectron quantum dot and the middle electron. (e) Calculated leakage spectrum, extracted from the calculated energy diagram as described in Sec. IV. (f) Dependence of the exchange energy extracted from the leakage spectroscopy in (c). (g) Leakage spectroscopy in a magnetic field applied perpendicular to the 2DEG plane, $B_{\perp}$. 
interaction with the middle spin has a positive sign, and therefore the transfer of the middle electron to the multielectron dot would result in a reduction of the ground state spin (from spin-1 to spin-1/2).

Insight into this regime can be gained from the Hubbard model, by calculating the energy spectrum associated with four electrons using Eq. (3). Figure 13(b) shows the result for a choice of input parameters that mimics the phenomenology of the multielectron device $\left(t_{1}=26 \mu \mathrm{eV}, t_{2}=12 \mu \mathrm{eV}\right.$, $t_{\mathrm{DD}}=30 \mu \mathrm{eV}$, and $\Delta E=40 \mu \mathrm{eV}$ ). As before, inspection of this energy diagram allows us to associate spin states with all eigenstates and infer the expected leakage spectrum from various state crossings. In particular, we associate the fielddependent state crossings arising in this energy diagram (marked by a white and black triangle) with the sharp leakage features observed in Fig. 13(c).

On the left-hand side of the diagram the multielectron dot is decoupled from the double dot by a large negative detuning voltage, and can be viewed as a spin-1 spectator spin. Thus, the eigenstates are tensor products of doubledot states $\left(|S\rangle,\left|T_{i}\right\rangle\right.$ with $\left.i=+,-, 0\right)$ and spin-1 states with different spin projections in the direction of the magnetic field $(|0\rangle$ or $| \pm 1\rangle)$. The three states that diverge towards small energies for large negative $\varepsilon^{*}$ all involve the doubledot singlet state $(|S\rangle)$, whereas the other states involve the double-dot triplet states. Therefore, the state plotted in blue can be viewed as a singletlike state, whereas the state in red is tripletlike.

In contrast, for large $\varepsilon^{*}$, the left dot decouples and becomes a spin- $1 / 2$ spectator, while the middle dot (with spin-1/2) hybridizes with the multielectron dot (spin-1) due to tunnel coupling. Accordingly, each eigenstate is the tensor product of a spin-1/2 state and one out of six "molecular states" formed by the middle and right dot. In terms of spin, these six "molecular states" comprise four quadruplet states $\left(\left|Q_{ \pm 3 / 2}\right\rangle,\left|Q_{ \pm 1 / 2}\right\rangle\right)$ with a total spin 3/2, and two doublet states $\left(\left|D_{ \pm 1 / 2}\right\rangle\right)$ with a total spin of $1 / 2$ (the subscript in our notation indicates the spin projection in the magnetic field direction). For doublet states the middle electron can relocate into the multielectron dot for large positive $\varepsilon^{*}$. Therefore, all four tensor products that involve doublet states diverge towards low energy in this regime. In contrast, spin-3/2 states within the multielectron dot would be costly in terms of single-particle energies, and hence, tensor products that involve the quadruplet states have associated with them a relatively stiff $(1,1, K)$ charge distribution, and transition into $(1,0, K+1)$ only for larger $\varepsilon^{*}$.

We believe that in the experiment we initialize the triple dot in the $|S ;+1\rangle$ state (in analogy to $|S ; \uparrow\rangle$ for the spin-1/2 multielectron dot). With increasing detuning this eigenstate continuously changes into $\left|\uparrow ; D_{1 / 2}\right\rangle$ [blue line in Fig. 13(b)]. This change of eigenstates explains the presence of exchange oscillations when pulsing $\varepsilon$ diabatically [Fig. 13(c)]. Meanwhile, the sharp features in the leakage spectrum correspond to the crossing of this blue colored state with a fully polarized $\left|T_{+} ;+1\right\rangle \equiv\left|\uparrow ; Q_{3 / 2}\right\rangle$ state [black and white triangles in Figs. 13(b) and 13(c)]. Indeed, the leakage spectrum inferred from the calculated energy spectrum confirms this analysis [Fig. 13(e)].

Finally, we present leakage spectroscopy measurements for the out-of-plane magnetic field $B_{\perp}$ [Fig. 13(e)]. Curiously, in this case we observe an additional leakage feature with a weaker dependence on detuning. At $\zeta \approx 18 \mathrm{mV}$, i.e., near the boundary between $(2,0,2 N+2)$ and $(1,1,2 N+2)$, this feature appears to cross the primary feature (which we associate with the $S-T_{+}$crossing within the double dot) without any sign of interaction. On the other hand, at the transition between $(1,1,2 N+2)$ and $(1,0,2 N+3)$ (higher detuning of the multielectron dot), the primary leakage feature ends at exactly the point where the additional feature crosses $\left(\varepsilon \approx 13 \mathrm{mV}, B_{\perp} \lesssim 180 \mathrm{mT}\right)$. We speculate that the additional feature arises from the strong coupling of multielectron-dot orbitals to the out-of-plane magnetic field, which breaks the near degeneracy between two orbitals [106] and drives a transition of the ground-state spin from $1\left(B_{\perp} \lesssim 180 \mathrm{mT}\right)$ to $0\left(B_{\perp} \gtrsim 180 \mathrm{mT}\right)$. This would explain the termination of the primary leakage feature at $\varepsilon \approx 13 \mathrm{mV}$ (since we know from Sec. IV that the primary leakage feature is absent when the multielectron quantum dot has a spin-0 ground state) as well as the absence of any interaction near $\zeta \approx 18 \mathrm{mV}$ (since the double dot in this low detuning is essentially decoupled from the multielectron dot).

\section{SUMMARY AND OUTLOOK}

We apply two methods developed for spin qubits to study the spin properties of a mesoscopic multielectron quantum dot, namely, spin leakage spectroscopy and measurement of coherent exchange oscillations. Both methods rely on fast gate-voltage pulses, single-shot charge detection, and spin-to-charge conversion, and provide complementary information about the multielectron dot (namely, incoherent spin leakage and coherent spin exchange processes). This allows us, for the first time, to study the spin spectrum associated with the multielectron dot (containing of order 100 electrons) and its dependence on the parity and charge occupation of the multielectron dot. By studying in detail the interaction of the multielectron dot with a neighboring electron (which we entangle with an unpaired reference electron prior to each pulse cycle), we discover a counterintuitive exchange profile between the neighboring electron and the odd-occupied multielectron quantum dot. Specifically, we observe that the exchange interaction rapidly varies with detuning voltages applied to the multielectron dot, resulting in local maxima and sign changes of the exchange interaction that can be controlled by few-millivolt changes in gate voltages. We also study even occupations of the multielectron dot, including a configuration with spin-1 ground state. We explain our 
observations using a Hubbard model, and classify its predicted exchange profiles into four distinct regimes. Using realistic parameters, we show that this model qualitatively reproduces the observed diverse behavior of the multielectron dot.

The key conclusion of this work is that a multielectron quantum dot possesses properties that may be exploited as a mediator of exchange interactions for spin qubit applications. We observe a spin-0 ground state, most desirable for long-range exchange coupling, for 3 out of 4 of the studied even occupancies. This should provide sufficient reliability for use in a scalable quantum dot system-accidental spin-1 ground states of the multielectron mediator can be avoided by adding or removing two electrons. A first demonstration towards long-range exchange coupling was recently demonstrated in the same device [55].

Several other findings may also benefit spin qubit applications. First, the appearance of extrema in the observed exchange profiles may be suitable to increase gate fidelities, by reducing the sensitivity of exchange oscillations to charge noise $[25,26]$. Second, access to both signs of the exchange lifts constraints for the construction of dynamically decoupled gates. Previous theoretical work assumed an exchange coupling that can only assume zero and positive values, and the resulting gate sequences can be quite complex $[107,108]$. Third, since the large quantum dot is characterized by a reduced level spacing, it may be possible to define a singlet-triplet rotating frame on the multielectron dot that is charge-noise insensitive (as demonstrated in Ref. [104]) but does not suffer from impractically high operating frequencies (analogous to the quantum dot hybrid qubit [5,9]). Fourth, a larger size of the multielectron quantum dot implies a reduction of the Overhauser field experienced by the electrons, and therefore a reduced dephasing rate [109]. Fifth, the addition of the single electron to the spin- 0 quantum dot preserves the spin of the electron. Therefore, it should be possible to subsequently eject this electron to another quantum dot, yielding a method for the coherent shuttling of spin states between distant quantum dots [110].

From a fundamental physics point of view, several aspects of the multielectron quantum dot can be studied further. For example, the distribution of level spacings and the strength of the spin correlation energy are likely characterized by mesoscopic fluctuations, and were not studied here. Their dependence on the dot size is of fundamental and practical importance. Further, our observation of spin-1 ground states suggests that spin-3/2 ground states should also occur, arguably with even higher probability [70,75]. Another curiosity is that for all three spin-1/2 ground states for which the quality of the data allowed a full analysis (i.e., $K=2 N-3,2 N-1,2 N+1$ ), we observe extrema in the exchange strength, both for positive and negative exchange strengths. This may hint towards a correlation between the level spacing and the ratio of the tunnel couplings.
Finally, for the first time we apply leakage spectroscopy and exchange-oscillation measurements of a spin qubit to study the spectrum of a more complicated, largely unknown object (the multielectron dot). The same principle could be applied to study numerous other systems and poorly understood phenomena. Examples include quantum dots coupled to quantum Hall or fractional quantum Hall edge states $[111,112]$, or to hybrid super-semiconducting quantum dots such as Majorana islands [113,114]. Going beyond one multielectron dot, we note that the combination of spin-1 states with the possibility to control the sign of exchange coupling between them may allow the exploration of exotic physics in multidot systems: Antiferromagnetically interacting spin-1 particles in a one-dimensional spin chain exhibit an energy gap and fractionalized edge mode degrees of freedom associated with the Haldane phase [115], which constitutes a canonical example of a symmetry-protected topologically ordered material [116,117]. Engineering such a spin chain in a system with the control and readout of quantum dots opens the possibility of exploring the long-range entanglement [118] of this topological phase and its potential use in quantum computing applications [119]. A scanning probe version of our technique, in which a spin qubit is scanned over surfaces as in a scanning tunneling microscope, would open the study of exchange interactions to an even larger class of quantum materials.

\section{ACKNOWLEDGMENTS}

We thank E. Barnes for helpful discussions. This work was supported by the Army Research Office, the Innovation Fund Denmark, the Villum Foundation, and the Danish National Research Foundation. Work at Sydney was supported by the ARC via the Centre of Excellence in Engineered Quantum Systems (EQuS), Project No. CE110001013. Work at Purdue was supported by the U.S. Department of Energy, Office of Basic Energy Sciences, Division of Materials, Sciences and Engineering under Award No. DE-SC0006671. Additional support from Nokia Bell Labs for the GaAs MBE effort is also gratefully acknowledged.

F. K. M. and F. M. contributed equally to this work.

\section{APPENDIX: PARAMETERS USED TO CALCULATE THE PRESENTED ENERGY SPECTRA}

The Hamiltonians Eqs. (2) and (3) contain parameters that were not measured directly, but which were estimated based on realistic experimental assumptions. These include the on- and off-site Coulomb interaction energies $U_{i}$ and $K_{i j}$ and the spin correlation energy $\xi$. To reduce the number of parameters in our modeling, we also fix certain combinations of single-particle energies, namely, $\bar{\varepsilon}=\left(\varepsilon_{L}+\varepsilon_{M}+\varepsilon_{R(1)}\right) / 3$ and $\varepsilon_{M}=\varepsilon_{M}-\left(\varepsilon_{L}+\varepsilon_{R(1)}\right) / 2$. 
All energy diagrams presented in this paper were calculated using identical sets of parameters, summarized in Table II, but differed in the assumed number of occupied orbitals appropriate for the different charge occupations of the multielectron dot.

[1] K. C. Nowack, M.Shafiei, M. Laforest, G. E. D. K. Prawiroatmodjo, L. R. Schreiber, C. Reichl, W. Wegscheider, and L. M. K. Vandersypen, Single-Shot Correlations and Two-Qubit Gate of Solid-State Spins, Science 333, 1269 (2011).

[2] M. D. Shulman, O. E. Dial, S. P. Harvey, H. Bluhm, V. Umansky, and A. Yacoby, Demonstration of Entanglement of Electrostatically Coupled Singlet-Triplet Qubits, Science 336, 202 (2012).

[3] L. Gaudreau, G. Granger, A. Kam, G. C. Aers, S. A. Studenikin, P. Zawadzki, M. Pioro-Ladrière, Z. R. Wasilewski, and A.S. Sachrajda, Coherent Control of Three-Spin States in a Triple Quantum Dot, Nat. Phys. 8, 54 (2012).

[4] S. A. Studenikin, G. C. Aers, G. Granger, L. Gaudreau, A. Kam, P. Zawadzki, Z. R. Wasilewski, and A. S. Sachrajda, Quantum Interference between Three Two-Spin States in a Double Quantum Dot, Phys. Rev. Lett. 108, 226802 (2012).

[5] G. Cao et al., A Tunable Hybrid Qubit in a GaAs Double Quantum Dot, Phys. Rev. Lett. 116, 086801 (2016).

[6] F. K. Malinowski et al., Notch Filtering the Nuclear Environment of a Spin Qubit, Nat. Nanotechnol. 12, 16 (2017).

[7] B. Bertrand, S. Hermelin, S. Takada, M. Yamamoto, S. Tarucha, A. Ludwig, A. D. Wieck, C. Bäuerle, and T. Meunier, Fast Spin Information Transfer between Distant Quantum Dots Using Individual Electrons, Nat. Nanotechnol. 11, 672 (2016).

[8] B. M. Maune et al., Coherent Singlet-Triplet Oscillations in a Silicon-Based Double Quantum Dot, Nature (London) 481, 344 (2012).

[9] D. Kim et al., Quantum Control and Process Tomography of a Semiconductor Quantum Dot Hybrid Qubit, Nature (London) 511, 70 (2014).

[10] K. Eng et al., Isotopically Enhanced Triple-Quantum-Dot Qubit, Sci. Adv. 1, e1500214 (2015).

[11] E. Kawakami et al., Gate Fidelity and Coherence of an Electron Spin in a Si/SiGe Quantum Dot with Micromagnet, Proc. Natl. Acad. Sci. U.S.A. 113, 11738 (2016).

[12] K. Takeda et al., A Fault-Tolerant Addressable Spin Qubit in a Natural Silicon Quantum Dot, Sci. Adv. 2, e1600694 (2016).

[13] M. Veldhorst et al., An Addressable Quantum Dot Qubit with Fault-Tolerant Control Fidelity, Nat. Nanotechnol. 9, 981 (2014).

[14] R. Maurand et al., A CMOS Silicon Spin Qubit, Nat. Commun. 7, 13575 (2016).

[15] J. J. Pla, K. Y. Tan, J. P. Dehollain, W. H. Lim, J. J. L. Morton, D. N. Jamieson, A. S. Dzurak, and A. Morello, A Single-Atom Electron Spin Qubit in Silicon, Nature (London) 489, 541 (2012).
[16] J. T. Muhonen et al., Storing Quantum Information for 30 Seconds in a Nanoelectronic Device, Nat. Nanotechnol. 9, 986 (2014).

[17] S. Foletti, H. Bluhm, D. Mahalu, V. Umansky, and A. Yacoby, Universal Quantum Control of Two-Electron Spin Quantum Bits Using Dynamic Nuclear Polarization, Nat. Phys. 5, 903 (2009).

[18] P. Cerfontaine, T. Botzem, S. S. Humpohl, D. Schuh, D. Bougeard, and H. Bluhm, Feedback-Tuned Noise-Resilient Gates for Encoded Spin Qubits, arXiv:1606.01897.

[19] J. Medford, J. Beil, J. M. Taylor, E. I. Rashba, H. Lu, A. C. Gossard, and C. M. Marcus, Quantum-Dot-Based Resonant Exchange Qubit, Phys. Rev. Lett. 111, 050501 (2013).

[20] J. Medford, J. Beil, J. Taylor, S. D. Bartlett, A. C. Doherty, E. I. Rashba, D. P. DiVincenzo, H. Lu, A. C. Gossard, and C.M. Marcus, Self-Consistent Measurement and State Tomography of an Exchange-Only Spin Qubit, Nat. Nanotechnol. 8, 654 (2013).

[21] J. M. Nichol, L. A. Orona, S. P. Harvey, S. Fallahi, G. C. Gardner, M. J. Manfra, and A. Yacoby, High-Fidelity Entangling Gate for Double-Quantum-Dot Spin Qubits, npj Quantum Inf. 3, 3 (2017).

[22] M. Veldhorst et al., A Two-Qubit Logic Gate in Silicon, Nature (London) 526, 410 (2015).

[23] T. F. Watson et al., A Programmable Two-Qubit Quantum Processor in Silicon, Nature (London), DOI: 10.1038/ nature25766 (in press).

[24] D. M. Zajac, A. J. Sigillito, M. Russ, F. Borjans, J. M. Taylor, G. Burkard, and J. R. Petta, Resonantly Driven CNOT Gate for Electron Spins, Science 359, 439 (2018).

[25] F. Martins, F. K. Malinowski, P. D. Nissen, E. Barnes, S. Fallahi, G. C. Gardner, M. J. Manfra, C. M. Marcus, and F. Kuemmeth, Noise Suppression Using Symmetric Exchange Gates in Spin Qubits, Phys. Rev. Lett. 116, 116801 (2016).

[26] M. D. Reed et al., Reduced Sensitivity to Charge Noise in Semiconductor Spin Qubits via Symmetric Operation, Phys. Rev. Lett. 116, 110402 (2016).

[27] D. Loss and D. P. DiVincenzo, Quantum Computation with Quantum Dots, Phys. Rev. A 57, 120 (1998).

[28] G. Burkard, D. Loss, and D. P. DiVincenzo, Coupled Quantum Dots as Quantum Gates, Phys. Rev. B 59, 2070 (1999).

[29] T. Meunier, V. Calado, and L. Vandersypen, Efficient Controlled-Phase Gate for Single-Spin Qubits in Quantum Dots, Phys. Rev. B 83, 121403 (2011).

[30] D. P. DiVincenzo, D. Bacon, J. Kempe, G. Burkard, and K. B. Whaley, Universal Quantum Computation with the Exchange Interaction, Nature (London) 408, 339 (2000).

[31] D. Bacon, J. Kempe, D. A. Lidar, and K. B. Whaley, Universal Fault-Tolerant Quantum Computation on Decoherence-Free Subspaces, Phys. Rev. Lett. 85, 1758 (2000).

[32] J. Taylor, H.-A. Engel, W. Dür, A. Yacoby, C. M. Marcus, P. Zoller, and M. D. Lukin, Fault-Tolerant Architecture for Quantum Computation Using Electrically Controlled Semiconductor Spins, Nat. Phys. 1, 177 (2005).

[33] M. Stopa and C. M. Marcus, Magnetic Field Control of Exchange and Noise Immunity in Double Quantum Dots, Nano Lett. 8, 1778 (2008). 
[34] S. Mehl and D. P. DiVincenzo, Noise Analysis of Qubits Implemented in Triple Quantum Dot Systems in a Davies Master Equation Approach, Phys. Rev. B 87, 195309 (2013).

[35] A. C. Doherty and M. P. Wardrop, Two-Qubit Gates for Resonant Exchange Qubits, Phys. Rev. Lett. 111, 050503 (2013).

[36] S. Mehl and D. P. Divincenzo, Noise-Protected Gate for Six-Electron Double-Dot Qubit, Phys. Rev. B 88, 161408 (2013).

[37] S. Mehl and D. P. DiVincenzo, Inverted Singlet-Triplet Qubit Coded on a Two-Electron Double Quantum Dot, Phys. Rev. B 90, 195424 (2014).

[38] S. Mehl, Two-Qubit Pulse Gate for the Three-Electron Double Quantum Dot Qubit, Phys. Rev. B 91, 035430 (2015).

[39] M. P. Wardrop and A. C. Doherty, Characterization of an Exchange-Based Two-Qubit Gate for Resonant Exchange Qubits, Phys. Rev. B 93, 075436 (2016).

[40] A. Sala and J. Danon, Exchange-Only Singlet-Only Spin Qubit, Phys. Rev. B 95, 241303 (2017).

[41] C. Kloeffel and D. Loss, Prospects for Spin-Based Quantum Computing in Quantum Dots, Annu. Rev. Condens. Matter Phys. 4, 51 (2013).

[42] O. Klochan et al., Double-Layer-Gate Architecture for GaAs Quantum Dots in the Few Hole Regime, Nanotechnology 494, 155329 (2015).

[43] D. M. Zajac, T. M. Hazard, X. Mi, E. Nielsen, and J. R. Petta, Scalable Gate Architecture for a One-Dimensional Array of Semiconductor Spin Qubits, Phys. Rev. Applied 6, 054013 (2016).

[44] G. Burkard and A. Imamoglu, Ultra-Long-Distance Interaction between Spin Qubits, Phys. Rev. B 74, 041307(R) (2006).

[45] Y.-Y. Liu, K. D. Petersson, J. Stehlik, J. M. Taylor, and J. R. Petta, Photon Emission from a Cavity-Coupled Double Quantum Dot, Phys. Rev. Lett. 113, 036801 (2014).

[46] J. J. Viennot, M. C. Dartiailh, A. A. Cottet, and T. Kontos, Coherent Coupling of a Single Spin to Microwave Cavity Photons, Science 349, 408 (2015).

[47] X. Mi, J. V. Cady, D. M. Zajac, P. W. Deelman, and J. R. Petta, Strong Coupling of a Single Electron in Silicon to a Microwave Photon, Science 355, 156 (2017).

[48] M. Russ and G. Burkard, Long Distance Coupling of Resonant Exchange Qubits, Phys. Rev. B 92, 205412 (2015).

[49] V. Srinivasa, J. M. Taylor, and C. Tahan, Entangling Distant Resonant Exchange Qubits via Circuit Quantum Electrodynamics, Phys. Rev. B 94, 205421 (2016).

[50] L. E. Bruhat, T. Cubaynes, J. J. Viennot, M. C. Dartiailh, M. M. Desjardins, A. Cottet, T. Kontos, and L. P. Aigrain, Strong Coupling between an Electron in a Quantum Dot Circuit and a Photon in a Cavity, arXiv:1612.05214.

[51] X. Mi, M. Benito, S. Putz, D. M. Zajac, J. M. Taylor, G. Burkard, and J. R. Petta, A Coherent Spin-Photon Interface in Silicon, arXiv:1710.03265.

[52] V. Srinivasa, H. Xu, and J. M. Taylor, Tunable Spin-Qubit Coupling Mediated by a Multielectron Quantum Dot, Phys. Rev. Lett. 114, 226803 (2015).
[53] S. Mehl, H. Bluhm, and D. P. DiVincenzo, Two-Qubit Couplings of Singlet-Triplet Qubits Mediated by One Quantum State, Phys. Rev. B 90, 045404 (2014).

[54] T. A. Baart, T. Fujita, C. Reichl, W. Wegscheider, and L. M. K. Vandersypen, Coherent Spin-Exchange via a Quantum Mediator, Nat. Nanotechnol. 12, 26 (2016).

[55] F. K. Malinowski, F. Martins, T. Smith, S. D. Bartlett, A. C. Doherty, P. D. Nissen, G. C. Gardner, S. Fallahi, M. J. Manfra, C. M. Marcus, and F. Kuemmeth, Fast Coherent Spin-Exchange via a Multielectron Quantum Mediator (to be published).

[56] J. A. Folk, S. R. Patel, S. F. Godijn, A. G. Huibers, S. M. Cronenwett, C. M. Marcus, K. Campman, and A.C. Gossard, Statistics and Parametric Correlations of Coulomb Blockade Peak Fluctuations in Quantum Dots, Phys. Rev. Lett. 76, 1699 (1996).

[57] L. P. Kouwenhoven, T. H. Oosterkamp, M. W. S. Danoesastro, M. Eto, D. G. Austing, T. Honda, and S. Tarucha, Excitation Spectra of Circular, Few-Electron Quantum Dots, Science 278, 1788 (1997).

[58] D. R. Stewart, D. Sprinzak, C. M. Marcus, C. I. Duruöz, and J.S. Harris, Jr., Correlations between Ground and Excited State Spectra of a Quantum Dot, Science 278, 1784 (1997).

[59] J. A. Folk, C. M. Marcus, R. Berkovits, I. L. Kurland, I. L. Aleiner, and B. L. Altshuler, Ground State Spin and Coulomb Blockade Peak Motion in Chaotic Quantum Dots, Phys. Scr. T90, 26 (2001).

[60] Y. Alhassid, The Statistical Theory of Quantum Dots, Rev. Mod. Phys. 72, 895 (2000).

[61] F. Martins, F. K. Malinowski, P. D. Nissen, S. Fallahi, G. C. Gardner, M. J. Manfra, C. M. Marcus, and F. Kuemmeth, Negative Spin Exchange in a Multielectron Quantum Dot, Phys. Rev. Lett. 119, 227701 (2017).

[62] X. G. Croot, S. J. Pauka, J. D. Watson, G. C. Gardner, S. Fallahi, M. J. Manfra, and D. J. Reilly, Device Architecture for Coupling Spin Qubits Via an Intermediate Quantum State, arXiv:1707.06479.

[63] X. Hu and S. D. Sarma, Spin-Based Quantum Computation in Multielectron Quantum Dots, Phys. Rev. A 64, 042312 (2001).

[64] C. Buizert, F. H. L. Koppens, M. Pioro-Ladrière, H.-P. Tranitz, I. T. Vink, S. Tarucha, W. Wegscheider, and L. M. K. Vandersypen, In Situ Reduction of Charge Noise in GaAs $/ \mathrm{Al}_{x} \mathrm{Ga}_{1-x} \mathrm{As}$ Schottky-Gated Devices, Phys. Rev. Lett. 101, 226603 (2008).

[65] J. Yoneda, T. Otsuka, T. Nakajima, T. Takakura, T. Obata, M. Pioro-Ladrière, H. Lu, C. J. Palmstrøm, A. C. Gossard, and S. Tarucha, Fast Electrical Control of Single Electron Spins in Quantum Dots with Vanishing Influence from Nuclear Spins, Phys. Rev. Lett. 113, 267601 (2014).

[66] J. R. Petta, A. C. Johnson, J. Taylor, E. A. Laird, A. Yacoby, M. D. Lukin, C. M. Marcus, M. P. Hanson, A. C. Gossard, and M. D. Lukin, Coherent Manipulation of Coupled Electron Spins in Semiconductor Quantum Dots, Science 309, 2180 (2005).

[67] D. J. Reilly, J. M. Taylor, E. A. Laird, J. R. Petta, C. M. Marcus, M. P. Hanson, and A. C. Gossard, Measurement of Temporal Correlations of the Overhauser Field in a 
Double Quantum Dot, Phys. Rev. Lett. 101, 236803 (2008).

[68] H. Bluhm, S. Foletti, D. Mahalu, V. Umansky, and A. Yacoby, Enhancing the Coherence of a Spin Qubit by Operating It as a Feedback Loop that Controls Its Nuclear Spin Bath, Phys. Rev. Lett. 105, 216803 (2010).

[69] S. Datta, Electronic Transport in Mesoscopic Systems (Cambridge University Press, Cambridge, England, 1997).

[70] P. W. Brouwer, Y. Oreg, and B. I. Halperin, Mesoscopic Fluctuations of the Ground-State Spin of a Small Metal Particle, Phys. Rev. B 60, R13977 (1999).

[71] I. L. Kurland, I. L. Aleiner, and B. L. Altshuler, Mesoscopic Magnetization Fluctuations for Metallic Grains Close to the Stoner Instability, Phys. Rev. B 62, 14886 (2000).

[72] H. U. Baranger, D. Ullmo, and L. I. Glazman, Interactions and Interference in Quantum Dots: Kinks in CoulombBlockade Peak Positions, Phys. Rev. B 61, R2425 (2000).

[73] Y. M. Blanter, A. D. Mirlin, and B. A. Muzykantskii, Fluctuations of Conductance Peak Spacings in the Coulomb Blockade Regime: Role of Electron-Electron Interaction, Phys. Rev. Lett. 78, 2449 (1997).

[74] K. Hirose and N. S. Wingreen, Ground-State Energy and Spin in Disordered Quantum Dots, Phys. Rev. B 65, 193305 (2002).

[75] H. Jiang, H. U. Baranger, and W. Yang, Spin and Conductance-Peak-Spacing Distributions in Large Quantum Dots: A Density Functional Theory Study, Phys. Rev. Lett. 90, 026806 (2003).

[76] U. Sivan, R. Berkovits, Y. Aloni, O. Prus, A. Auerbach, and G. Ben-Yoseph, Mesoscopic Fluctuations in the Ground State Energy of Disordered Quantum Dots, Phys. Rev. Lett. 77, 1123 (1996).

[77] K. Deng, N. J. Mayhall, and E. Barnes, Negative Exchange Interactions in Coupled Few-Electron Quantum Dots, arXiv:1712.05795.

[78] S. Tarucha, D. G. Austing, T. Honda, R. J. van der Hage, and L. P. Kouwenhoven, Shell Filling and Spin Effects in a Few Electron Quantum Dot, Phys. Rev. Lett. 77, 3613 (1996).

[79] D. M. Zumbühl, C. M. Marcus, M. P. Hanson, and A. C. Gossard, Cotunneling Spectroscopy in Few-Electron Quantum Dots, Phys. Rev. Lett. 93, 256801 (2004).

[80] D. A. Gorokhov and P. W. Brouwer, Combined Effect of Electron-Electron Interactions and Spin-Orbit Scattering in Metal Nanoparticles, Phys. Rev. B 69, 155417 (2004).

[81] The mechanisms underlying Hund's rule have been analyzed primarily in few-electron systems with high spatial symmetries, i.e., atoms and artificial atoms (small circular quantum dots), where degenerate electronic shells are generic $[77,82,83,105]$. For a small, interacting, disordered system, subjecting a restricted version of the Hartree-Fock approximation to the rules of random matrix theory yields on-site (Hubbard) interaction terms that compete against the kinetic energy cost associated with a finite level spacing [70]. Although the orbital energy splitting and exchange energy between any two orbitals are random, a general interaction Hamiltonian emerges in the limit of large Thouless conductance, which depends only on the mean exchange energy and mean level spacing (and an attractive interaction in the Cooper channel for the case of superconducting instability). Similar to the Stoner instability, their relative ratio determines the probability for such a dot to display mesoscopic magnetism, i.e., the distribution function of high-spin ground states within an ensemble of dots $[71,75,80]$. One may view the shift of this distribution function (towards higher values of total spin) just below the Stoner instability as a mesoscopic analog of Hund's rule.

[82] R. J. Boyd, A Quantum Mechanical Explanation for Hund's Multiplicity Rule, Nature (London) 310, 480 (1984).

[83] J. Cioslowski, K. Strasburger, and E. Matito, Benchmark Calculations on the Lowest-Energy Singlet, Triplet, and Quintet States of the Four-Electron Harmonium Atom, J. Chem. Phys. 141, 044128 (2014).

[84] A. V. Andreev and A. Kamenev, Itinerant Ferromagnetism in Disordered Metals: A Mean-Field Theory, Phys. Rev. Lett. 81, 3199 (1998).

[85] S. Lindemann, T. Ihn, T. Heinzel, W. Zwerger, K. Ensslin, K. Maranowski, and A. C. Gossard, Stability of Spin States in Quantum Dots, Phys. Rev. B 66, 195314 (2002).

[86] E. A. Laird, J. R. Petta, A. C. Johnson, C. M. Marcus, A. Yacoby, M. P. Hanson, and A. C. Gossard, Effect of Exchange Interaction on Spin Dephasing in a Double Quantum Dot, Phys. Rev. Lett. 97, 056801 (2006).

[87] C. Barthel, D. J. Reilly, C. M. Marcus, M. P. Hanson, and A. C. Gossard, Rapid Single-Shot Measurement of a Singlet-Triplet Qubit, Phys. Rev. Lett. 103, 160503 (2009).

[88] C. Barthel, J. Medford, H. Bluhm, A. Yacoby, C. M. Marcus, M. P. Hanson, and A. C. Gossard, Relaxation and Readout Visibility of a Singlet-Triplet Qubit in an Overhauser Field Gradient, Phys. Rev. B 85, 035306 (2012).

[89] M. D. Shulman, S. P. Harvey, J. M. Nichol, S. D. Bartlett, A. C. Doherty, V. Umansky, and A. Yacoby, Suppressing Qubit Dephasing Using Real-Time Hamiltonian Estimation, Nat. Commun. 5, 5156 (2014).

[90] M. R. Delbecq et al., Quantum Dephasing in a Gated GaAs Triple Quantum Dot due to Nonergodic Noise, Phys. Rev. Lett. 116, 046802 (2016).

[91] F. K. Malinowski, F. Martins, L. Cywiński, M. S. Rudner, P. D. Nissen, S. Fallahi, G. C. Gardner, M. J. Manfra, C. M. Marcus, and F. Kuemmeth, Spectrum of the Nuclear Environment for GaAs Spin Qubits, Phys. Rev. Lett. 118, 177702 (2017).

[92] For example, from measurements performed for a specific odd occupancy, say $K$, the assumptions of an associated spin- $1 / 2$ or spin-3/2 ground state yield different predictions for the expected presence or absence of a spinful ground state at occupancy $K+1$, and its associated exchange profile, which we can verify experimentally.

[93] F. Simmel, T. Heinzel, and D. A. Wharam, Statistics of Conductance Oscillations of a Quantum Dot in the Coulomb-Blockade Regime, Europhys. Lett. 38, 123 (1997).

[94] S. R. Patel, S. M. Cronenwett, D. R. Stewart, A. G. Huibers, C. M. Marcus, C. I. Duruöz, J. S. Harris, K. Campman, and A.C. Gossard, Statistics of Coulomb Blockade Peak Spacings, Phys. Rev. Lett. 80, 4522 (1998). 
[95] Conventionally, the terms "singlet" and "triplet" are used for two-electron spin-singlet states and two-electron spintriplet states, and one may be tempted to use these terms as synonyms for the symmetry under exchange of two spins. Care must be taken when giving in to this temptation for three-electron states, as their total antisymmetry arises in general from a combination of orbital and spin symmetries. In this work, we therefore use "singletlike" and "tripletlike" only for states that can be written as a product state of one spectator spin $(|\uparrow\rangle$ or $|\downarrow\rangle)$ and a two-electron spin state, which may be a spin-singlet state $(S)$ or one of three possible spin-triplet states $\left(T_{+}, T_{0}, T_{-}\right)$. Similarly, fourelectron states relevant for Sec. VI behave under certain circumstances like the tensor product of a spin-1 spectator (located in the right dot) and a singlet or triplet twoelectron spin state within the left dot middle dot double dot.

[96] E. A. Laird, J. M. Taylor, D. P. DiVincenzo, C. M. Marcus, M. P. Hanson, and A. C. Gossard, Coherent Spin Manipulation in an Exchange-Only Qubit, Phys. Rev. B 82, 075403 (2010).

[97] G. Poulin-Lamarre, J. Thorgrimson, S. A. Studenikin, G. C. Aers, A. Kam, P. Zawadzki, Z. R. Wasilewski, and A. S. Sachrajda, Three-Spin Coherent Oscillations and Interference, Phys. Rev. B 91, 125417 (2015).

[98] F. K. Malinowski, F. Martins, P. D. Nissen, S. Fallahi, G. C. Gardner, M. J. Manfra, C. M. Marcus, and F. Kuemmeth, Symmetric Operation of the Resonant Exchange Qubit, Phys. Rev. B 96, 045443 (2017).

[99] J. M. Taylor, V. Srinivasa, and J. Medford, Electrically Protected Resonant Exchange Qubits in Triple Quantum Dots, Phys. Rev. Lett. 111, 050502 (2013).

[100] M. Russ and G. Burkard, Asymmetric Resonant Exchange Qubit under the Influence of Electrical Noise, Phys. Rev. B 91, 235411 (2015).

[101] M. Russ, F. Ginzel, and G. Burkard, Coupling of ThreeSpin Qubits to Their Electric Environment, Phys. Rev. B 94, 165411 (2016).

[102] C. Zhang, X.-C. Yang, and X. Wang, Leakage and Sweet Spots in Triple-Quantum-Dot Spin Qubits: A Molecular Orbital Study, arXiv:1711.06418.

[103] M. Russ and G. Burkard, Three-Electron Spin Qubits, J. Phys. Condens. Matter 29, 393001 (2017).

[104] O. E. Dial, M. D. Shulman, S. P. Harvey, H. Bluhm, V. Umansky, and A. Yacoby, Charge Noise Spectroscopy Using Coherent Exchange Oscillations in a Singlet-Triplet Qubit, Phys. Rev. Lett. 110, 146804 (2013).

[105] Y. Sajeev, M. Sindelka, and N. Moiseyev, Hund's Multiplicity Rule: From Atoms to Quantum Dots, J. Chem. Phys. 128, 061101 (2008).
[106] A. Hofmann et al., Measuring the Degeneracy of Discrete Energy Levels Using a GaAs/AlGaAs Quantum Dot, Phys. Rev. Lett. 117, 206803 (2016).

[107] X. Wang, L. S. Bishop, J. P. Kestner, E. Barnes, K. Sun, and S. D. Sarma, Composite Pulses for Robust Universal Control of Singlet-Triplet Qubits, Nat. Commun. 3, 997 (2012).

[108] X. Wang, L. S. Bishop, E. Barnes, J. P. Kestner, and S. D. Sarma, Robust Quantum Gates for Singlet-Triplet Spin Qubits Using Composite Pulses, Phys. Rev. A 89, 022310 (2014).

[109] J. M. Taylor, J. R. Petta, A. C. Johnson, A. Yacoby, C. M. Marcus, and M. D. Lukin, Relaxation, Dephasing, and Quantum Control of Electron Spins in Double Quantum Dots, Phys. Rev. B 76, 035315 (2007).

[110] T. Fujita, T. A. Baart, C. Reichl, W. Wegscheider, and L. M. K. Vandersypen, Coherent Shuttle of Electron-Spin States, npj Quantum Inf. 3, 22 (2017).

[111] G. Yang, C.-H. Hsu, P. Stano, J. Klinovaja, and D. Loss, Long-Distance Entanglement of Spin Qubits via Quantum Hall Edge States, Phys. Rev. B 93, 075301 (2016).

[112] H. Kiyama, T. Nakajima, S. Teraoka, A. Oiwa, and S. Tarucha, Spin-Dependent Current through a Quantum Dot from Spin-Polarized Nonequilibrium Quantum Hall Edge Channels, Phys. Rev. B 91, 155302 (2015).

[113] K. Gharavi, D. Hoving, and J. Baugh, Readout of Majorana Parity States Using a Quantum Dot, Phys. Rev. B 94, 155417 (2016).

[114] M. T. Deng, S. Vaitiekènas, E. B. Hansen, J. Danon, M. Leijnse, K. Flensberg, J. Nygård, P. Krogstrup, and C. M. Marcus, Majorana Bound State in a Coupled QuantumDot Hybrid-Nanowire System, Science 354, 1557 (2016).

[115] F. D. M. Haldane, Nonlinear Field Theory of Large-Spin Heisenberg Antiferromagnets: Semiclassically Quantized Solitons of the One-Dimensional Easy-Axis Néel State, Phys. Rev. Lett. 50, 1153 (1983).

[116] Z.-C. Gu and X.-G. Wen, Tensor-Entanglement-Filtering Renormalization Approach and Symmetry-Protected Topological Order, Phys. Rev. B 80, 155131 (2009).

[117] X. Chen, Z.-C. Gu, and X.-G. Wen, Classification of Gapped Symmetric Phases in One-Dimensional Spin Systems, Phys. Rev. B 83, 035107 (2011).

[118] L. C. Venuti and M. Roncaglia, Analytic Relations between Localizable Entanglement and String Correlations in Spin Systems, Phys. Rev. Lett. 94, 207207 (2005).

[119] D. V. Else, I. Schwarz, S. D. Bartlett, and A. C. Doherty, Symmetry-Protected Phases for Measurement-Based Quantum Computation, Phys. Rev. Lett. 108, 240505 (2012). 